\title{
TERAHERTZ BAND COMMUNICATIONS AS A NEW FRONTIER FOR DRONE NETWORKS
}

\author{
Akhtar Saeed ${ }^{1}$, Ozgur Gurbuz ${ }^{1}$, Mustafa Alper Akkaş ${ }^{2}$, Ahmet Ozan Bicen ${ }^{3}$ \\ ${ }^{1}$ Electronics Engineering Program, Faculty of Engineering and Natural Sciences, Sabanci University, Istanbul, 34956, \\ Turkey, ${ }^{2}$ Department of Computer Engineering, Bolu Abant Izzet Baysal University, Bolu, 14280, Turkey, ${ }^{3}$ Independent \\ Contributor
}

NOTE: Corresponding author: Akhtar Saeed, akhtarsaeed@sabanciuniv.edu

\begin{abstract}
Terahertz band (0.1-10 THz) communications is one of the candidates for $6 \mathrm{G}$ systems due to intrinsic massive bandwidth and data rate support. Having demonstrated the significant potential of THz band at various atmospheric altitudes, in this article, we discuss the prospects of THz communications for drone networks, more specifically, for Drone Sensor Networks (DSNs). For $6 G$ non-terrestrial communication scenarios, drones will not only serve as on-demand base-stations, as supporting alternatives or backhauls for the terrestrial base stations, but they will also provide seamless connectivity for distributed monitoring and surveillance applications, which require an ultra-reliable low latency service for carrying multimedia data. THz band sensing will also provide additional sensing capabilities from the sky to THz-enabled DSNs. Presenting this vision, in this paper, we first discuss possible use cases of THz-enabled drone networks considering communication, sensing and localization aspects. Then, for revealing the capacity potential of THz-enabled drone networks, we provide motivating channel capacity results for communication of drones at different altitudes, under ideal channel conditions with no fading and realistic channel with beam misalignment and multipath fading. We further present major challenges pertaining to employing the THz band for DSNs, addressing physical layer issues, followed with spectrum and interference management, medium access control and higher layers and security, while reviewing some prominent solutions. Finally, we highlight future research directions with Artificial Intelligence (AI)/Machine Learning (ML)-based approaches and mobile edge computing.
\end{abstract}

Keywords - Artificial intelligence, disaster management, drone networks, drone sensor networks, machine learning, mobile edge computing, monitoring, surveillance, terahertz communications, terahertz sensing

\section{INTRODUCTION}

Drones will soon inhabit our skies as they are easily available, reliable and low-cost devices. The demand for such hovering drones is increasingly witnessed in civil and government applications, as globally, many governments and industries have been investing heavily in deploying drone networks as per their requirements [1]. Typically, small drones with multi-copter-like functionalities are favo-rable due to their cheap maintenance and convenient deployments [2]. In order to achieve a certain mission, it is usually desirable to deploy a collection or swarm of drones in a networked fashion [3]. Such drone networks or Drone Sensor Networks (DSNs) can monitor a large coverage area and the sensed data can be gathered with enhanced reliability, resilience and fault tolerance under diverse conditions.

DSNs can be highly viable in many real-world scenarios: For military surveillance applications (Fig. 1(a)), DSNs can monitor a sensitive area, such as across international borders, where highly delicate military data (in the form of images or videos) can be transmitted securely. In addition to the communications perspective, DSNs involving drones with sensing and processing capabilities can be utilized in some applications, such as disaster management, for instance for detecting dangerous gases (Fig. 1(b)). Moreover, drone networks with drones with base station-like capabilities can provide seamless on- demand network coverage from the air (Fig. 1(c)), as an alternative as well as support to the terrestrial base stations, for communal gatherings, concerts etc. Such drone base stations can also be useful in disaster-struck areas, where the terrestrial communication infrastructure is damaged. Nevertheless, in each of the above-mentioned possible real-world applications, frequent mobility of the drones with constrained energy resources will be required to be addressed to achieve the desired/optimal performance.

The intelligent information society of 2030 is expected to be globally information driven, highly digitized, with the support of unlimited and near instant complete wireless connectivity [4]. 6G, here, will be the prime catalyst for achieving this target, connecting everything, including wireless coverage in all dimensions, as well as concatenating almost all different functions such as, communication, sensing, imaging, computing, caching, navigation (e.g., radars), control, for supporting nearly all real-world applications [5]. As wireless communications are rapidly progressing towards $6 \mathrm{G}$, from the exponentially growing network traffic arises need of exploiting the electromagnetic spectrum above the existing sub $6 \mathrm{GHz}$ bands, which are almost saturated. A possible solution to this need is to utilize the Terahertz (THz) band $(0.1-10 \mathrm{THz})$ [6], as the bridge between the $5 \mathrm{G}$ millimeter wave band and the free space optics band [7, 8]. A THz band offers huge bandwidth, favorable for very high data rate 


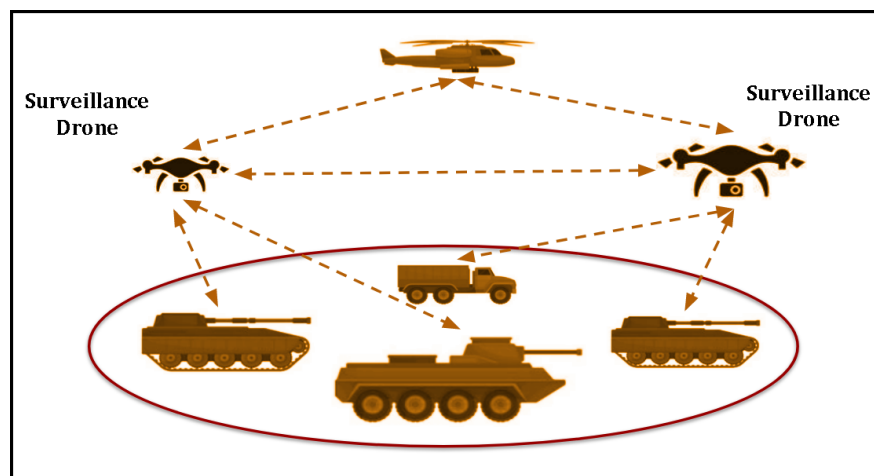

(a) Military Surveillance

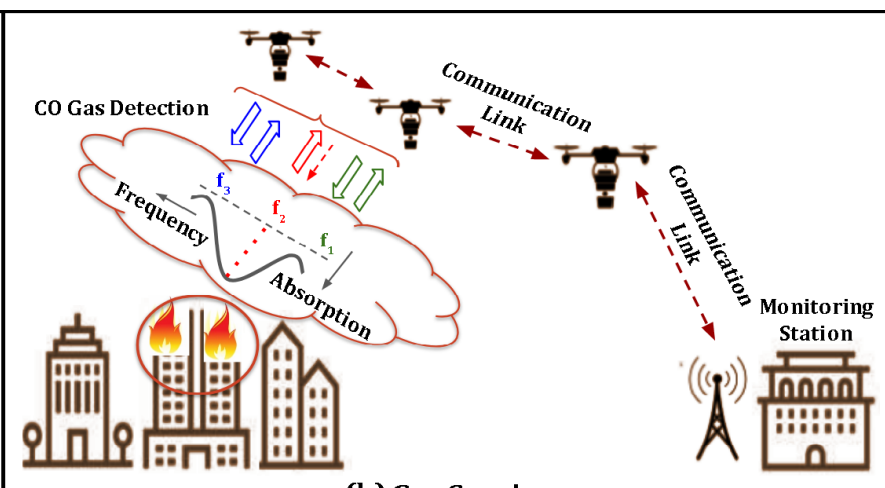

(b) Gas Sensing

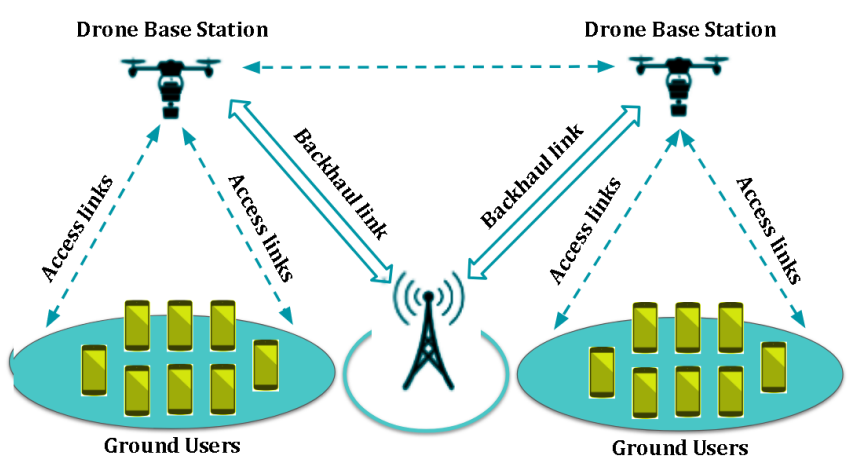

(c) On-Demand Coverage With Drone Base Station

Fig. 1 - Possible real-world scenarios of THz-enabled drone networks.

applications, while at the same time promises massive antenna gains due to shorter wavelengths $[9,10]$. However, $\mathrm{THz}$ communications are restricted by the absorption loss, which is highest at the sea-level, as the atmospheric gas concentration is at the maximum [11, 12]. Consequently, $\mathrm{THz}$ communications have been studied mainly for short transmission distances at the sea-level, such as for on- chip communications [13] or for connecting data centers within up to $10 \mathrm{~m} \mathrm{[14].}$

$\mathrm{THz}$ bands have been recently considered for aerial communications. Despite the highly mobile nature of aerial vehicles, a THz band, due to very high frequency, promises minimized Doppler effect, making massive rate communication links realizable within mobile aerial vehicles by optimal selection of the beam patterns [15]. For the razor-sharp beams due to carrier frequencies in the order of $\mathrm{THz}$, the communication links between hovering aerial vehicles have to be highly aligned. The influence of the micro, small and large-scale mobility uncertainties for drones communicating in millimeter wave and $\mathrm{THz}$ bands are studied in [16, 17]. It is shown that without adaptive beam-width control, micro-scale mobility induces negligible link capacity degradation, whereas small-scale mobility and large-scale mobility can induce significant degradation in the link capacity, with larger outages. THz-based drone (Unmanned Aerial Vehicle, UAV) networks are analyzed in [18] by assessing coverage probability and area spectrum efficiency. It is concluded that due to massive path loss incurred by THz band waves at $0.35 \mathrm{THz}$, a larger UAV density is required for a certain coverage probability, as compared to lower carrier frequencies. In [19], THz MIM0-OFDM communication between two UAVs is studied by analyzing the orientation and position estimation error bounds. It is shown that the positioning accuracy at the millimeter levels can be achieved provided that the transmitter to receiver separation is considerably small.

In [11], we have performed path loss and total usable bandwidth analyses over a THz band (0.75-10 THz), considering constant narrowband noise approach for four types of aerial vehicles at different altitudes: Drones (at $1 \mathrm{~km}$ ), jet planes (at $10 \mathrm{~km}$ ), high altitude UAVs (at $16 \mathrm{~km}$ ), and satellites (at $99 \mathrm{~km}$ ). The path loss analysis shows that the absorption effect diminishes at the higher altitudes and the total path loss behaves as free space spread loss. Moreover, the total usable bandwidth analysis infers that at the higher altitudes (e.g. high altitude UAVs and satellites), the entire $\mathrm{THz}$ band $(0.75-10 \mathrm{THz}$ ) becomes feasible as a single transmission window, which is about 9.25 THz wide. In our subsequent work [12], we have proposed an alternative channel model for THz communications, where, by taking the colored nature of noise Power Spectral Density (PSD) into account, the commonly flat bands in path loss (gain) and noise PSD are determined for the THz spectrum $(0.75-10 \mathrm{THz})$, and a variable bandwidth capacity computation method is proposed as an alternative to the standard capacity computation. Our extensive capacity analysis of the same four aerial 
scenarios has shown that under fading channel conditions, the ergodic capacity at the sea-level is enhanced by an order of magnitude for the drones hovering at $100 \mathrm{~m}$ altitude supporting multiple Tbps at a range of $10 \mathrm{~m}$, whereas $10 \mathrm{~s}$ of Tbps are realizable among jet planes (10 $\mathrm{km}$ altitude) and the high altitude UAVs $(16 \mathrm{~km}$ altitude), and multitude of $100 \mathrm{~s}$ of Tbps are also achievable for inter-satellite links (also cubesats) at a range of $1 \mathrm{~km}$. In both works [11, 12], it is concluded that the $\mathrm{THz}$ communications is highly viable for non-terrestrial communications. Motivated by our earlier analyses for $\mathrm{THz}$ communications among aerial vehicles in $[11,12]$, in this survey, we review $\mathrm{THz}$ band communications and sensing for drone networks, more specifically for DSNs and we highlight the prospects. First, we present applications of THz-enabled DSNs. Secondly, we present capacity results for drone-to- drone links, considering drones at different altitudes and varying fading conditions. We show that $\mathrm{THz}$ communi- cations can be quite promising even under realistic fading conditions, providing up to many Tbps at $10 \mathrm{~m}$ range and $10 \mathrm{~s}$ of Gbps at $50 \mathrm{~m}$ range. Motivated by the capacity potential, we next discuss the open issues for THz communications in DSNs, along with research directions. Lastly, we highlight Artificial Intelligence (AI) and Machine Learning (ML)-based approaches.

\section{APPLICATIONS OF THZ BAND DRONE NETWORKS}

With the $\mathrm{THz}$ band being one of the key enablers of prospective $6 \mathrm{G}$ research, we envision employing the $\mathrm{THz}$ band for drone networks as expected use cases of 6G non-terrestrial networks. Fig. 2 depicts such possible applications of THz-enabled drone networks, namely monitoring and surveillance, sensing, localization and on- demand network coverage, as elaborated next. It is worth-mentioning here that within each of the following applications of the $\mathrm{THz}$ band communications, sensing and localization, it will be important to deploy the THz- enabled drones strategically [6] so as to fully exploit the $\mathrm{THz}$ band for drone networks and DSNs. These strate- gies include Ultra-Massive Multiple Input Multiple Out- put (UM-MIMO) and Reconfigurable Intelligent Surfaces (RIS), which are explained in Section 4 of this paper, in detail.

\subsection{Monitoring/Surveillance}

As 6G research is progressing towards ubiquitous automation, aerial monitoring using drones has become increasingly popular [20]. Networked state-of-the-art drones will become an essential aerial resource for many real-world monitoring applications, such as military surveillance, disaster management, etc. In the following, we cover such practical monitoring applications for THz-enabled drone networks.

\section{Military Applications}

Drone networks have become an integral part of military applications around the globe. With the aid of quickly deployable drone networks, a multitude of military-related activities can be performed effectively. Such activities include border patrolling by monitoring high resolution real-time videos. For instance in [21], the concept of BorderSense is introduced, which is a new hybrid concept of wireless sensor networks, including underground sensors, on-ground sensors, and drones for wireless sensing from the air. With the BorderSense framework, drones can provide mobility freedom together with on-board high resolution cameras and highly sensitive sensors for provisioning an enhanced coverage as per the military requirements. Here, for the military applications, bandwidth should be provisioned substantially together with an ultra-reliable low latency service in order to transfer sensitive military data/information within fractions of a second [10]. In addition, drones providing such military applications (e.g., air to air and air to ground) will need to be highly energy efficient as it will not be realistically possible to frequently replace their on-board power sources (batteries). With THz-enabled DSNs, a massive THz bandwidth will promise high resolution video monitoring of sensitive areas, as in BorderSense framework. Moreover, thanks to the unique propagation characteristics of the $\mathrm{THz}$ band, $\mathrm{THz}$ waves show a unique reflective nature to the metallic surfaces. Therefore, a $\mathrm{THz}$ band can be effectively utilized in the military surveillance applications such as detecting certain weapons [22]. The detection of any possible explosives is also possible using $\mathrm{THz}$ spectral imaging [23]. For instance, $\mathrm{THz}$ spectral imaging can detect small land mines, while the ground radars cannot distinguish between such mines and rocks [24]. THz-enabled DSNs with mobility freedom can assist a military by detecting such mines without any direct human contact.

\section{Disaster Management}

Drone networks or DSNs can be considered in various disaster management applications, e.g., for Early Warning Systems (EWSs), which perform environmental and structural monitoring and process the collected informa- tion for disaster predictions. Such a DSN can also support Search And Rescue (SAR) missions in the post disaster management scenarios. DSNs can be utilized to restore the damaged communication infrastructure in case of a disaster. Additionally, post-disaster damage assessment can be made possible using DSNs with video monitoring [25]. THz-enabled drone networks and DSNs with massive bandwidth can support an aerial communication backup for EWS, SAR over the disaster-struck region. Energy efficiency of the drones will be a critical parameter for such autonomous aerial operations [26], where frequent replacement of the on-board batteries may not be a feasible operation. 


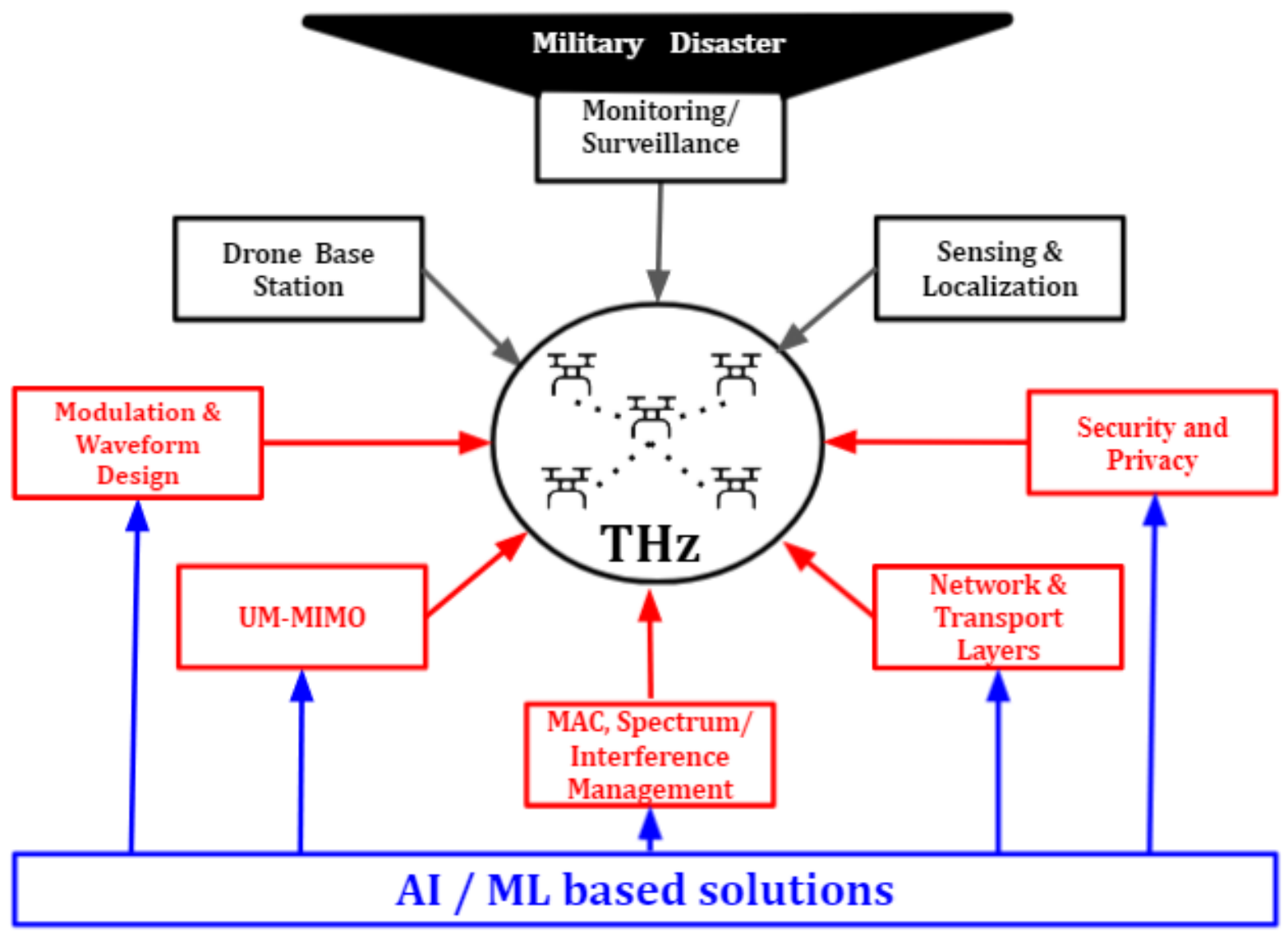

Fig. 2 - THz-enabled drone sensor networks: Applications, challenges, and solutions.

\subsection{Terahertz for sensing}

Unlike infrared and microwaves, THz waves hold many unique transmission properties such as a quasi-optical nature and molecular absorption, making the $\mathrm{THz}$ band an appropriate candidate for some sensing applications, such as gas and material sensing, quality control, chemical and biological sensing, bearing unique $\mathrm{THz}$ spectral signatures. Hence, THz-enabled DSNs can be deployed in a variety of practical sensing applications. $\mathrm{THz}$ signals possess distinctive spectral signatures, i.e., quasi-optical nature having varying absorption loss across the $\mathrm{THz}$ band, due to the molecular rotations/ vibrations, promising that $\mathrm{THz}$ waves can be highly suitable for applications related to the rotational spectroscopy, such as gas detection $[6,22]$. Consequently, it becomes possible to utilize $\mathrm{THz}$ waves for the purposes of detecting various poisonous/dangerous gases like hydrogen cyanide, carbon monoxide, etc., possibly over disaster-struck places, including fire eruption, massive earthquakes, volcanic eruptions, etc. THz-enabled DSNs, here, can be employed to promptly detect/sense such poisonous gases with the aid of $\mathrm{THz}$ waves by rapid detection and relaying such vital information, saving any further victims and avoiding other secondary disasters. Both active and passive sensing methods can be employed for poisonous gas detection using $\mathrm{THz}$ spectroscopy.
An example of detecting a dangerous gas, such as carbon monoxide is illustrated in Fig. 1. Here, a THz wave with spectrum ranging from $f_{1}$ to $f_{3}$ is transmitted through a drone $\mathrm{Tx}$, which is then received by the drone $\mathrm{Rx}$. If the dangerous gas pertaining an absorption at $f_{2}$ is available, the received $\mathrm{THz}$ wave level at $f_{2}$ shall be lower as compared to the other frequencies. Thus, the gaseous presence is sensed/detected. For the gas sensing, $\mathrm{THz}$ band of $0.2-1 \mathrm{THz}$ has been considered in the literature [27]. After detecting the gas successfully, again, the $\mathrm{THz}$ band can be utilized for relaying (communicating) the sensed information via drone networks to a nearby monitoring station for rapid rescue operations. Thus, an all-in-one operation (sensing and communication) can be conveniently achieved by deploying THz-enabled DSNs and drone networks.

\subsection{Localization}

Besides other requirements of the prospective 6G systems, high precision localization is also expected. It is predicted that the $\mathrm{THz}$ band will promise localization accuracy in the order of centimeters [28], which is far better than the existing localizationbased ser- vices, such as Global Positioning System (GPS), and cell- multilateration. Localization methods at higher frequencies, such as across the $\mathrm{THz}$ band rely on the technique of Simultaneous Localization and Mapping 
(SLAM), where the overall accuracy is enhanced by obtaining very high resolution images of the surroundings (environment). SLAM techniques comprise of three main stages: 1) image capturing of the surroundings, 2) range estima- tion from the user, and 3) aggregation of the images at the approximated ranges. For example, an accuracy of the sub-centimeter levels can be acquired by making 3- dimensional images of the surroundings with the aid of $\mathrm{THz}$ signals and projecting the time and angle of arrival details from the user for estimating the locations. Instead of ground-based localization, THz-enabled DSNs can assist localization applications with an improved aerial field of view over a larger coverage area [22].

\subsection{Drone base stations}

The concept of Drone Base Stations (DBS) has recently emerged in the literature, e.g., [29, 30, 31, 32], where a hovering/mobile drone with base station-like capabilities can serve the ground users as an alternative back-up to the terrestrial network. DBS has already been investigated in [33] as an extension of 5G. However, there is some recent work on DBS for $6 \mathrm{G}$ networks and tech- nologies. For instance in [7], DBSs have been considered as a subset of Wireless Infrastructure Devices (WIDs), promising coverage and capacity improvements for the prospective 6G networks. In [34], DBSs have been recognized as a major challenge related to the intelligent handover of drones in multiple DBS networks for 6G technologies. By deploying DBS, aerial wireless coverage can be provided to the ground users as well as to the hot spots, especially in the areas/regions with scarce or no communication infrastructure [35]. Also, it will be possible to provide seamless high rate connectivity for realtime multimedia streaming and/or for on-demand applications, such as in concerts [36,37], reducing the overall communication load of a nearby terrestrial base station. Moreover, in case of a natural disaster, multiple DBSs can be deployed over the entire disaster-struck area with the damaged communication infrastructure, e.g., macro-hot spots [29], thereby providing high rate communication backup from the air. Here, for DBS scenarios, bandwidth should be adequately provisioned with an ultra-low latency service to provide uninterrupted coverage to the ground users. Thanks to a large THz bandwidth, THzenabled DBSs can support each of the above use cases effectively. Still, THz-enabled DBSs will also take into account frequent handovers between adjacent DBSs for providing seamless coverage to the ground users [34].

\section{CAPACITY OF THZ COMMUNICATION AMONG DRONES}

Having discussed the insights into the possible applications of the THz-enabled drone networks, in this section, we present $\mathrm{THz}$ channel capacity analysis and some results for practical drone scenarios as our motivation for considering THz communications in DSNs.

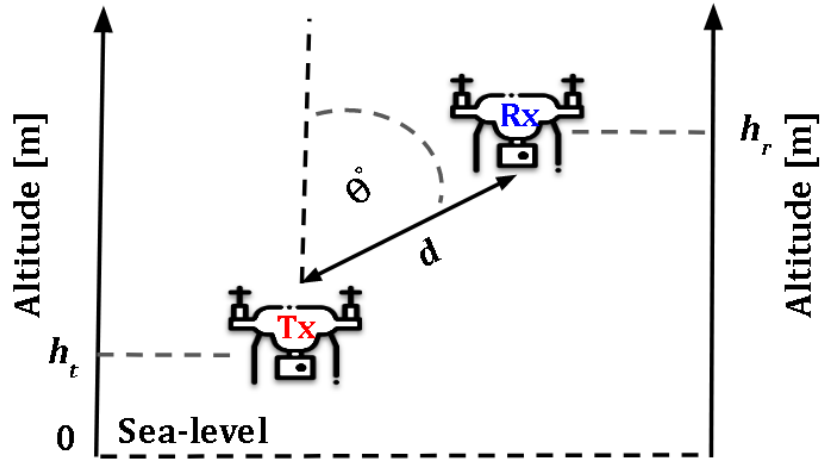

Fig. 3 - System model of a THz-enabled drone network at various atmospheric altitudes consisting of a transmitter drone at altitude $h_{t}$, and a receiver drone at altitude, $h_{r}$. For a given $h_{t}, h_{r}$ is found according to $\theta$ and $d$.

Fig. 3 illustrates a simple system model, where a transmit- ter drone, $\mathrm{Tx}$, and a receiver drone, $\mathrm{Rx}$ are hovering at alti- tudes $h_{t}$ and $h_{r}$, respectively. $\theta$ is the phase from the verti- cal axis, determining the direction of communication. For instance, $\theta=0^{\circ}$ corresponds to the vertically-up communi- cation, $90^{\circ}$ denotes horizontal direction, while $180^{\circ}$ is the vertically-down direction. As discussed in Section 1, THz waves experience a dominant water vapor absorption gain in addition to the free space spread gain. Besides, the Beam Misalignment (BM) fading and Multipath (MP) fading also contribute to the overall gain obtained as follows $[12,38]$ :

$$
G=G_{l} G_{p} G_{f},
$$

where, $G_{l}$ is the path gain coefficient, and $G_{p}$ and $G_{f}$ refer to BM and MP fading, respectively. $G_{l}$ includes the free space spread gain coefficient, $G_{s}$, and the absorption gain coefficient, $G_{a}$ given as:

$$
G_{l}\left(f, h_{t}, h_{r}, d\right)=G_{l}\left(f, h_{t}, \theta, d\right)=G_{s}(f, d) G_{a}\left(f, h_{t}, \theta, d\right),
$$

where $f$ denotes the carrier frequency of the THz wave in hertz, $h_{t}, h_{r}$ are the transmitter (Tx) and receiver (Rx) altitudes in meters, respectively. $d$ is the transmission distance between Tx and $\mathrm{Rx}$, and $\theta$ denotes the relative position of Rx with respect to Tx as depicted in Fig. 3. $G_{s}(f, d)$ is the free space spread gain coefficient due to the $\mathrm{THz}$ wave attenuation as it propagates across the atmosphere via an isotropic antenna, obtained as:

$$
G_{s}(f, d)=\frac{c}{4 \pi f d},
$$

where $c$ is the speed of the THz wave in free space, i.e., $299,792,458 \mathrm{~m} / \mathrm{s}$.

$G_{a}\left(f, h_{t}, \theta, d\right)$, the absorption gain coefficient is mainly induced by the water vapor molecules present in the atmosphere, mathematically expressed as,

$$
G_{a}\left(f, h_{t}, \theta, d\right)=\left(\tau\left(f, T\left(h_{t}, \theta, d\right), v\left(h_{t}, \theta, d\right)\right)\right)^{1 / 2}
$$


where $\tau\left(f, T\left(h_{t}, \theta, d\right), v\left(h_{t}, \theta, d\right)\right)$ is the atmospheric medium's transmittance. We employ Line-By-Line Radiative Transfer Model (LBLRTM) for obtaining realistic transmittance values across various atmospheric altitudes $[39,40] . T\left(h_{t}, \theta, d\right)$ and $v\left(h_{t}, \theta, d\right)$ are the atmospheric temperature (in Kelvin) and water vapor concentration (in \%), respectively, for the link between the Tx drone hovering at $h_{t}$ and $\mathrm{Rx}$ drone at $h_{r}$.

In regard to the impact of the mobility of the communicating drones and the resulting Doppler spread, thanks to the very high operating frequency in the order of $\mathrm{THz}$, mobile drones observe minimized Doppler effect [15], promising high rate links between communicating drones. It has been shown in $[41,42]$ that for a typical drone relative velocity, $v_{r}=10 \mathrm{~m} / \mathrm{s}$, the maximum Doppler shift is negligible. As an example, by considering $f_{c}=0.75 \mathrm{THz}$, and $v_{r}=10 \mathrm{~m} / \mathrm{s}$, the maximum Doppler shift is: $f_{d . \max }=f_{c} \cdot v / c=25017.31 \mathrm{~Hz}$, which is negligible in terms of the inter-carrier interference. Moreover, since we are con- sidering drone to drone communications, where a swarm (group) of drones move together, $v_{r}$ can be less than $10 \mathrm{~m} / \mathrm{s}$ (up to $0 \mathrm{~m} / \mathrm{s}$ ) and $f_{d . \max }$ will be even smaller than the example provided above. Therefore, we neglect the effect of the Doppler spread in our capacity computations.

For beam misalignment fading, we consider the following probability density function for the BM fading coefficient, $G_{p}$, [43]:

$$
f_{G_{p}}(x)=\frac{\zeta^{2}}{A_{0}^{\zeta^{2}}} x^{\zeta^{2}-1}
$$

where $\zeta=\frac{w_{e q}}{2 \sigma_{s}}, w_{e q}$ is the equivalent $\mathrm{Tx}$ beam width, $\sigma_{s}$ denotes the jitter (BM) standard deviation, and $A_{0}$ is the fraction of power collected at Rx at no beam misalignment. This BM fading model has been widely employed in many studies of free space optical systems. For more details of the BM fading model, we refer to our earlier work [12] and also [43].

Finally, for incorporating multipath fading, we consider the famous $\alpha-\mu$ model as follows [44]:

$$
f_{G_{f}}(x)=\frac{\alpha \mu^{\mu}}{\hat{G}_{f}^{\alpha \mu} \Gamma(\mu)} x^{\alpha \mu-1} \exp \left(-\mu \frac{x^{\alpha}}{\hat{G}_{f}^{\alpha}}\right),
$$

where $f_{G_{f}}(x)$ is the pdf of the MP fading coefficient, $G_{f}$, $\alpha$ is the fading parameter, $\mu$ is the normalized variance of the channel envelope under fading, and $G_{f}$ is the $\alpha$-root mean value. The $\alpha-\mu$ model is a common model of several famous fading distributions. For instance, $\alpha=2$ and $\mu=1$ represents Rayleigh fading, etc.

For computing noise power, $P_{n}$, we consider a constant narrowband approach [14] across the $\mathrm{THz}$ band (0.75-10 THz), where each narrowband, $\Delta f$ is $0.3 \mathrm{GHz}$ wide, which is the spectral resolution of LBLRTM. Numerically,

$$
P_{n}\left(f, h_{t}, \theta, d, \Delta f\right)=k_{B} \int_{\Delta f} T_{n o i s e}\left(f, h_{t}, \theta, d\right) d f,
$$

where $k_{B}$ is the Boltzmann's constant, $T_{\text {noise }}$ is the molecular noise temperature obtained as $T_{\text {noise }}\left(f, h_{t}, \theta, d\right)=$ $T_{0} \epsilon\left(f, h_{t}, \theta, d\right)$. Here, $T_{0}$ is the reference temperature (in Kelvin), and $\epsilon$ is the channel's emissivity, $\epsilon\left(f, h_{t}, \theta, d\right)=1$ - $\tau\left(f, T\left(h_{t}, \theta, d\right), v\left(h_{t}, \theta, d\right)\right)$ [14]. Hence, $T_{n o i s e}$ is a function of transmittance, $\tau$, which is obtained using LBLRTM. In the results provided in this paper, for capturing the absorption effect across the $\mathrm{THz}$ band $(0.75-10 \mathrm{THz})$, US Standard 1976 weather profile is set in LBLRTM [12]. For computing the capacity of $\mathrm{THz}$ drone-to-drone links under ideal, no fading channel, the total channel gain is set as, $G=G_{l}$. In this paper, we consider the standard narrowband capacity computation in $[14,45]$ :

$$
C\left(h_{t}, \theta, d\right)=\sum_{k=1}^{K} \Delta f \log _{2}\left[1+\frac{P_{T}^{k}\left|G\left(f_{k}, h_{t}, \theta, d\right)\right|^{2} G_{T}}{P_{n}\left(f_{k}, h_{t}, \theta, d,(\Delta f)\right)}\right],
$$

where total transmit power, $P_{T}$, and total antenna gain (from Tx and Rx antennas), $G_{T}$ are set to practical values as $P_{T}=24 \mathrm{dBm}(0.25 \mathrm{~W})[46]$ and $G_{T}=60 \mathrm{dBi}[47,48]$, respectively. For power allocation, we consider both the Water-Filling (WF) and Equal-Power (EP) schemes [14]. In WF allocation, the total transmit power, $P_{T}$ is optimally distributed across the $\mathrm{THz}$ band $(0.75-10 \mathrm{THz})$ comprising of constant narrowbands, $k=1,2,3, \ldots, K$, each $0.3 \mathrm{GHz}$ wide (i.e., LBLRTM's spectral resolution), as:

$$
\frac{P_{T}^{k}}{P_{T}}= \begin{cases}\frac{1}{\gamma_{\circ}}-\frac{1}{\gamma_{k}} & , \gamma_{k} \geq \gamma_{\circ} \\ 0 & , \gamma_{k}<\gamma_{\circ}\end{cases}
$$

where $P_{T}^{k}$ is the optimal power for the constant narrowband, $k, \gamma_{0}$ is the threshold SNR, $\gamma_{k}$ is the SNR of $k$. $\gamma_{\circ}$ is obtained by $\sum_{k=1}^{K}\left(\frac{1}{\gamma_{\circ}}-\frac{1}{\gamma_{k}}\right)=1$ [49].

In EP allocation, $P_{T}$ is distributed equally within all $k$ across the entire THz band (0.75-10 THz) [12].

For the channel under fading, involving BM and MP fading, the channel gain is set as $G=G_{l} G_{p} G_{f}$, and we evaluate the ergodic capacity by averaging results over 100 realizations, as follows:

$$
\overline{C\left(h_{t}, \theta, d\right)}=\Delta f \mathbb{E}\left(\sum_{k=1}^{K} \log _{2}\left[1+\frac{P_{T}^{k}\left|G\left(f_{k}, h_{t}, \theta, d\right)\right|^{2} G_{T}}{P_{n}\left(f_{k}, h_{t}, \theta, d,(\Delta f)\right)}\right]\right),
$$

where $\mathbb{E}($.$) denotes the expectation taking over channel$ realizations under fading.

Next, we present the capacity and ergodic capacity results specifically for drone scenarios, considering various practical settings of Tx and Rx drone altitudes, zenith angles and transmission ranges. Fig. 4(a)-(c) depict the channel capacity as the function of transmission range under ideal, i.e., under no fading channel. The capacity results with no fading (ideal) channel are included in our analysis as the benchmark to compare how much of the capacity is degraded when realistic beam misalignment fading and multipath fading are introduced into the channel, as provided in the subsequent discussion. Three drone Tx 
altitudes, $h_{t}$, are considered i.e., $h_{t}=100 \mathrm{~m}, 500 \mathrm{~m}$, and $1 \mathrm{~km}$, whereas $h_{r}$ i.e., the $\mathrm{Rx}$ drone altitude for each setting is obtained using $h_{t}, \theta$ (angle in degrees between Tx and Rx drones), and $d$.

It can be seen for a given $h_{t}$, changing $\theta$, i.e., the direction of communication from $0^{\circ}$ (vertically-up) (Fig. 4(a)) to $90^{\circ}$ (horizontal) (Fig. 4(b)) down to $180^{\circ}$ (vertically-down)(Fig. 4(c)) do not incur considerable variations in the ca- pacity. This is due to the dense and homogeneous atmosphere across lower atmospheric altitudes. Nevertheless, increasing $h_{t}$ shows promising capacity improvements. For instance, at $h_{t}=100 \mathrm{~m}, 0^{\circ}$, and $d=100 \mathrm{~m}$, capacity values correspond to $505.8 \mathrm{Gbps}$ and 34.52 Gbps with WF and EP allocation schemes, respectively. For the identical $\theta$ and $d$ settings but at a higher Tx drone altitude, $h_{t}=1 \mathrm{~km}$, the capacity values stand at $652.6 \mathrm{Gbps}$ and $62.89 \mathrm{Gbps}$ with WF and EP allocations, respectively. This is because traversing up across the atmosphere from $100 \mathrm{~m}$ to $1 \mathrm{~km}$ observes substantial decrements in the water vapor concentration levels [12], which can be highly leveraged in drone networks communicating over the $\mathrm{THz}$ band. Additionally, for overcoming the distance issue observed across lower atmospheric levels, for instance, for $h_{t}$ lower than $100 \mathrm{~m}$, multiple drones can be deployed sufficiently close to each other, in a networked fashion, where they can be treated as relays. These results showcase the massive capacity potential of the $\mathrm{THz}$ band for drone networks, promising links in the order of up to several 10s of Gbps using EP allocation, and up to many 100s of Gbps with WF power allocation for transmission ranges up to $100 \mathrm{~m}$. Fig. 5 depicts the ergodic capacity trend for short range, i.e., $d=10 \mathrm{~m}$, under BM fading and MP fading parameters [12]. Interestingly, it can be seen in Fig. 5(a) that for MP fading parameter, $\mu=1$, which corresponds to pure NLOS, Rayleigh fading, increasing the normalized jitter standard deviation, $\sigma_{s} / a$ does not cause substantial ergodic capacity degradation. Due to nearby reflections the NLOS MP fading components are more dominating than the BM fading components for short range; hence severeness of BM does not affect the ergodic capacity. Meanwhile, Fig. 5(b) shows that for fixed BM fading parameter, $\sigma_{s} / a=5$, MP fading degrades the ergodic capacity by $26 \%$ for EP allocation, and $16.5 \%$ for WF allocation, as the MP effect is varied from $\mu=10$, indicating a strong LOS along with NLOS components to $\mu=1$, i.e., pure NLOS Rayleigh fading. Next, we present the achievable ergodic capacity at $d=50 \mathrm{~m}$, under variable BM fading with Rayleigh MP fading ( $\mu=1$ ) in Fig. 6(a), and variable MP fading with $\sigma_{s} / a=5$ Fig. 6(b). For this range, increasing $\sigma_{s} / a$ from 1 to 10 decreases the ergodic capacity substantially, e.g., by an order of magnitude for EP allocation, as $\sigma_{s} / a$ is increased from 1 to 10 . On the other hand, decreasing $\mu$, from 10 to 1 with given $\sigma_{s} / a=5$ shows no considerable change in ergodic capacity. The ergodic capacity results in Fig. 5 and Fig. 6 depicting that at short ranges, it is the MP fading that mainly affects ergodic capacity, while at long range, it is mainly the BM fading. With this analysis, we emphasize that $\mathrm{THz}$ band communication in drone networks can promise massive rate links even under realistic BM fading and MP fading con- ditions. We refer the readers to [12] for an in-depth ca- pacity analysis of $\mathrm{THz}$ communications for drones and the other three aerial vehicles, where both standard narrowband and variable bandwidth capacity computa- tions are considered for various altitudes, distances, posi- tions/orientations of the vehicles (i.e., the entire range of $\theta$ from $0^{\circ}$ to $180^{\circ}$ ) by leveraging LBLRTM for THz absorp- tion gains, evaluating no fading, BM fading and MP fading conditions.

\section{OPEN ISSUES AND RESEARCH DIREC- TIONS}

Design and implementation of THz-enabled drone networks and DSNs require novel communication schemes and networking protocols, including but not limited to modulation and waveform design, ultra-massive Multiple Input Multiple Output (MIMO), spectrum and interference management, Medium Access Control (MAC) and higher network layers, security and privacy issues.

\subsection{Physical layer}

$\mathrm{THz}$ band drone communications primarily requires enhanced $\mathrm{THz}$ band channel models. For this purpose, measurement-based studies need to be pursued at drone altitudes in various propagation environments and under drone mobility scenarios, so that the existing line-of-sight and non line-of-sight models with beam misalignment and generic multipath fading (as considered in this work) can be improved with specific stochastic channel models for THz links among drones. A recent work on active and passive THz systems is presented in [50], where measurement results at $140 \mathrm{GHz}(0.14 \mathrm{THz})$ have been provided for rooftop surrogate satellite systems and terrestrial networks. Based on the enhanced channel models, modulation and waveform design should be tailored for $\mathrm{THz}$ band communications in drone networks or DSNs.

\section{Modulation}

The state-of-the-art modulation schemes that can be potentially employed for $\mathrm{THz}$ band communications include Single-Carrier (SC) modulation, multi-carrier modulation, Orthogonal Frequency Division Multiplexing (OFDM), Cyclic Prefix Orthogonal Frequency Division Multiplexing (CP-OFDM) and even Non-Orthogonal Multiple Access (NOMA). In what follows, we discuss each of the aforementioned modulation schemes in the perspective of $\mathrm{THz}$ band communications for drone networks. Non-overlapping transmission windows are termed as Single Carrier (SC) modulation, having some provision of the carrier aggregation [51]. However, due to the intrinsic frequency-selective nature of $\mathrm{THz}$ channel, multi-carrier modulations would also help in some form of carrier aggregation with multiple individual/ non-overlapping single carriers [52]. The implementation of practical $\mathrm{THz}$ transceivers is another challenging task, 


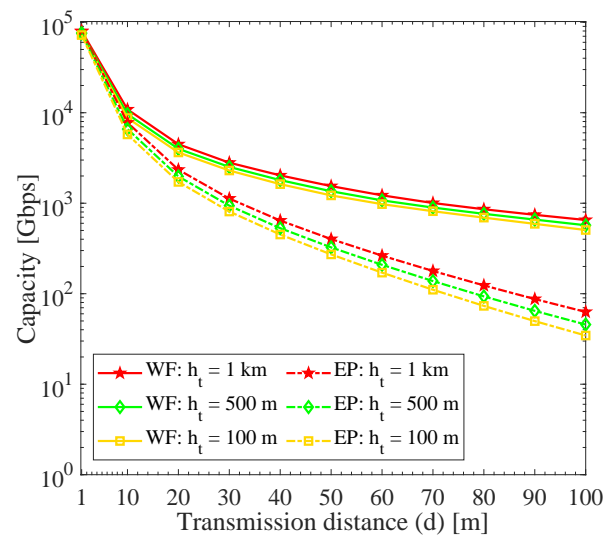

(a) Vertically-up communication, $\theta=0^{\circ}$.

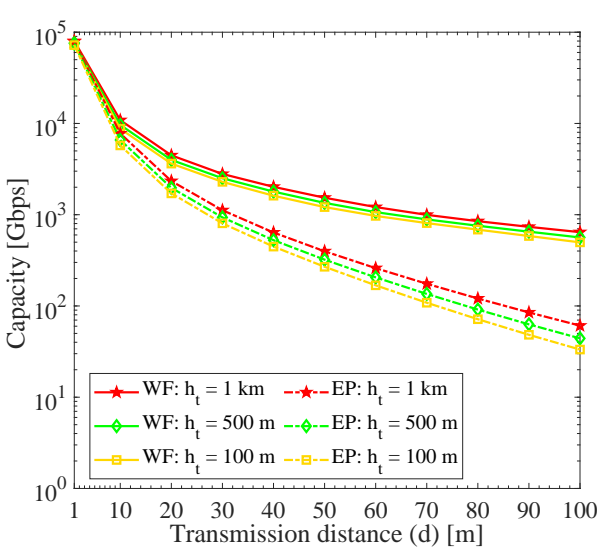

(b) Horizontal communication, $\theta=90^{\circ}$.

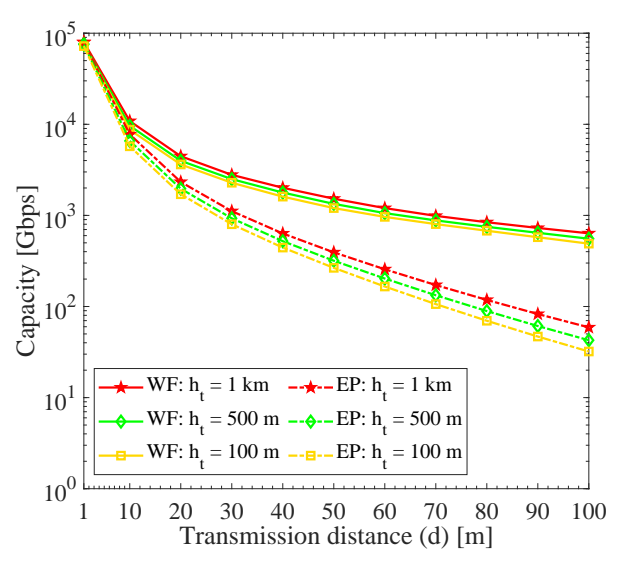

(c) Vertically-down communication, $\theta=180^{\circ}$.

Fig. 4 - Capacity under no fading as a function of distance, for various drone transmitter altitudes and directions of communication (vertically up, horizontal and vertically down, i.e., $\theta=0^{\circ}, 90^{\circ}$, and $180^{\circ}$, respectively). For a given $h_{t}$ and $d$, varying direction $(\theta)$ does not considerably affect the capacity, as the atmosphere is dense and homogeneous across for drone altitudes up to $h_{t}=1 \mathrm{~km}$.

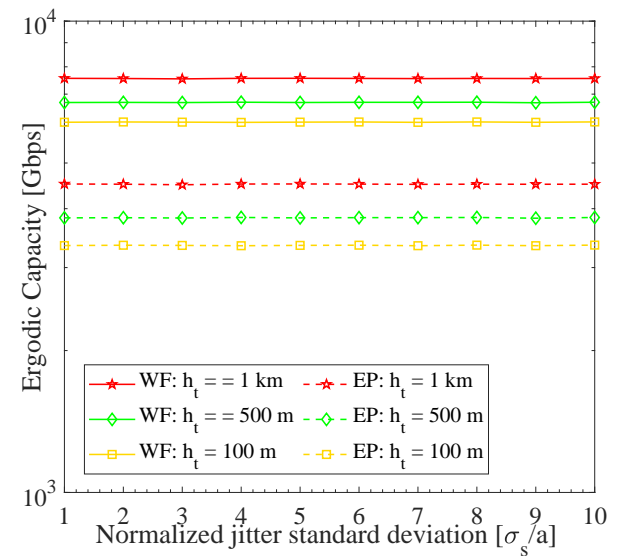

(a) MP fading parameter, $\mu=1$.

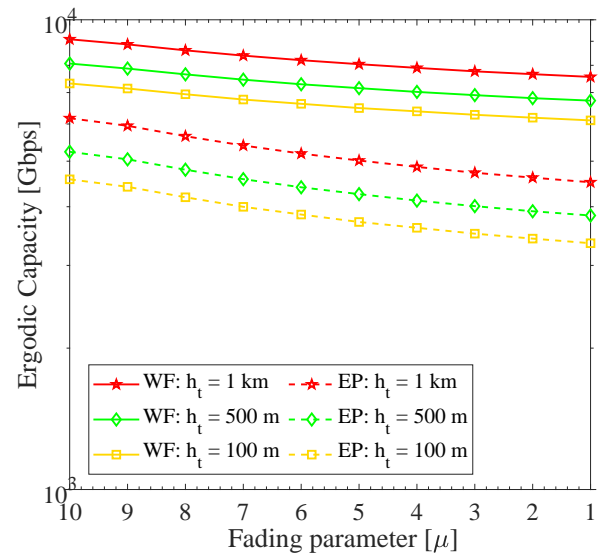

(b) Normalized jitter standard deviation, $\sigma_{s} / a=5$.

Fig. 5 - Effect of beam misalignment fading and multipath fading on the ergodic capacity: $\theta=90^{\circ}$ (horizontal communication), and $d=10 \mathrm{~m}$ across various drone transmitter altitudes. For this range, MP fading shows more dominant impact on the ergodic capacity as compared to BM fading due to short transmission distance.

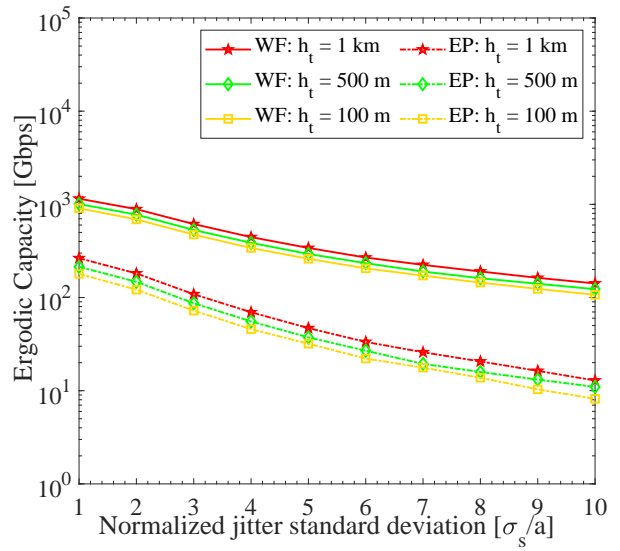

(a) MP fading parameter, $\mu=1$.

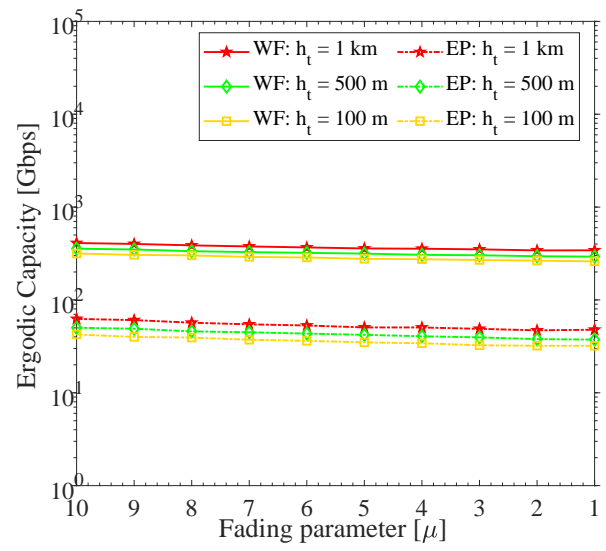

(b) Normalized jitter standard deviation, $\sigma_{s} / a=5$.

Fig. 6 - Effect of beam misalignment fading and multipath fading on the ergodic capacity: $\theta=90^{\circ}$ (horizontal communication), and $d=50 \mathrm{~m}$ across various drone transmitter altitudes. For this case, BM fading shows more dominant impact on the ergodic capacity as compared to the MP fading, due to a large transmission distance. 
as the conventional RF circuitry cannot support data rates in the order of several 100s of Gbps or Tbps as provisioned in Section 3. Moreover, novel signal processing techniques will be required to counter the mismatch between the state-of-the-art digital baseband systems and the large bandwidth offered by the $\mathrm{THz}$ band [52]. Recently, there have been advancements related to the $\mathrm{THz}$ transceivers; both in the electronic and the photonic domains [22]. The advancements towards practical $\mathrm{THz}$ transceivers (both electronic and photonic) have been well summarized in [53]. For short-range communication (below one meter), impulse-radio-like communication based on one-hundredfemtosecond-long pulses following an on-off keying modulation spread in time has been proposed in [36]. Such very short pulses, which are already utilized as the basis of many $\mathrm{THz}$ sensing systems, can be generated and detected with current technologies. For longer communication distances, new dynamic bandwidth modulations are required for not only overcoming but also leveraging the unique distance-dependent bandwidth created by molecular absorption [11, 12]. Orthogonal Frequency Division Multiplexing (OFDM) has widely been implemented in broadband wireless systems since 4G for achieving higher spectral efficiency. In [54], OFDM is proposed for $60 \mathrm{GHz}$ millimeter wave systems. For $5 \mathrm{G}$, several wireless standards including Long Term Evolution (LTE), Wireless Fidelity (Wi-Fi), Asynchronous Digital Subscriber Line (ADSL) etc., have adopted Cyclic Prefix Orthogonal Frequency Division Multiplexing (CP-OFDM)[55]. Multiple communicating nodes, each with Tbps of data (which would also be the case in THz-enabled DSNs) would require relaying data in an asynchronous manner. Also, in such asynchronous multiple user access, the subcarriers with CP-OFDM do not remain orthogonal, which introduces substantial inter-carrier interference [56]. This makes CP-OFDM infeasible for DSNs. Nevertheless, OFDM systems promise utilization of the nonoverlapping spectrum for the improved spectrum efficiency as compared to the pulse-based communication systems [57, 58]. However, implementation of OFDM transceivers in the $\mathrm{THz}$ band is especially complex due to stringent frequency synchronization requirements, with the sampling rates in the typical order of several Giga samples/sec or even Tera samples per second. Additionally, large high Peak-to-Average Power Ratio (PAPR) also makes OFDM implementation not feasible over the $\mathrm{THz}$ band [52, 59]. In recent years, Non-Orthogonal Multiple Access (NOMA) has gained considerable attention, as it promises not only greater link rates for both the downlink and uplink transmissions, but it also provisions a way to counter the packet collision issue, e.g., in MTC with grant-free access [60]. NOMA is adopted for THz systems in [61] by making use of the frequency and distancedependent $\mathrm{THz}$ spectrum. The concept of hybrid beamforming is proposed for forming user clusters, and NOMAbased grouping and Long-User-Central-Window (LUCW)based sub-band allotment within a user cluster are proposed for improving user fairness as well as spectral efficiency. For mitigating the water vapor-based absorption effect in the $\mathrm{THz}$ band, the conventional modulation schemes can be further optimized. For this purpose, in [51], distance-aware multi-carrier schemes are proposed. Resource optimizations include power allocation as in [57], where long range networks are established using a pulse-based multi-wide band waveform design by adapting power allocations over variable number of frames. By adapting the symbol time and modulation order, in [59], a hierarchical modulation scheme is proposed for a system with a single transmitter and multiple receivers, supporting various streams of data for multiple users at variable ranges. In [62], distance-adaptive and bandwidth-dependent modulations using OFDM in $\mathrm{THz}$ band are proposed. It is worth mentioning here that, the aforementioned schemes have been proposed for $\mathrm{THz}$ band communications at sea level. These schemes will need to be adapted for specifically the drone scenarios or DSNs, considering altitudes as well as drone mobility.

\section{Ultra-Massive MIMO}

A main issue for the $\mathrm{THz}$ band communications is constituted by the frequency-selective and an extremely high path loss, which simply crosses $100 \mathrm{~dB}$ for ranges greater than only a few meters under LOS channels. This path loss is even worse under NLOS channel conditions. Consequently, huge gains by highly directional antennas are required for communicating over ranges greater than a few meters. In this regard, the idea of Ultra-Massive (UM)-MIMO has been proposed [63], where extremelydense arrays of plasmonic nano-antennas are employed. In lieu of deploying the traditional metallic antennas, meta-materials and nano-materials can be exploited for manufacturing plasmonic nano-antennas, which are sufficiently less than the wavelength of the operating carrier frequency. This unique property of the plasmonic nanoantennas enables them to be packed in massively densed arrays. For instance, for an array with a footprint of $1 \mathrm{~mm} \times 1 \mathrm{~mm}$, a sum of 1024 plasmonic nano-antennas designed for $1 \mathrm{THz}$ carrier frequency can be integrated together, keeping the inter-element distance (spacing) of $1 / 2$ of the plasmonic wavelength. Similar arrays of the plasmonic nano-antennas can be employed at both the $\mathrm{Tx}$ and $\mathrm{Rx}$ sides simultaneously for countering the massive path loss issue by: 1) Overcoming the spread loss, by targeting the signal transmission in space, and 2) focusing the bandwidths for the signal transmission within the windows having the least absorption levels. By intelligently inputting the array elements, variations of the modes of operation can be utilized in an adaptive fashion. For instance, in UM-Beam-forming (UM-BF), all of the antennas utilize the identical transmit signal, similar to the case of conventional beam-forming. Such a mode can substantially counter the massive path loss/attenuation at the $\mathrm{THz}$ band carrier frequencies, thus supporting communication to larger ranges. Additionally, beam-forming also mitigates 
the problem of the co-channel interference, also utilizing the freedom of angle diversity by moving the razor-sharp $\mathrm{THz}$ beam to specific or targeted directions. Using UM-MIMO, a certain group of arrays/antenna elements can be designated for communicating to a specific user. This special mode utilizes various data streams onto a single carrier frequency, thereby increasing the per user capacity, which is also beneficial when the communication links are operating in a limited bandwidth and a high SNR scheme. This special mode enhances the rate by the virtue of Spatial Multiplexing (SM), provided that the channel matrix of the UM-MIMO has sufficient rank and diversity. Conclusively, any amalgamation across UM-SM and UM-BF is realizable. Additionally, for maximizing the utilization of the $\mathrm{THz}$ band, promising Tbps links, multiple transmission windows can be employed simul- taneously. For this purpose, multiband UM-MIMO utilizes different carrier frequencies by tuning electrically the frequency response of the plasmonic nano-antennas. One of the major pros of this multiband UM-MIMO technique is that the data can be processed within a substantially smaller bandwidth, hence, lowering the complexity of the system design with an improved flexibility of the spectrum. In this research arena, novel frequency, space and time modulation and coding methods are required to be proposed for such UM-MIMO communication systems. UM-MIMO can also be leveraged for DSNs due to very large beam-forming gains to overcome the huge path loss over the THz band. Moreover, as a byproduct, razor-sharp $\mathrm{THz}$ beams would substantially mitigate the interference among communicating drones in the DSNs. Nevertheless, the design of UM-MIMO systems for DSNs and drone networks should also incorporate the effect mobility of the drones to avoid Tx-Rx antenna beam misalignment and maximize the beam-forming. For the case of DBS, implementation of UM-MIMO will be essential to provide aerial coverage to several users at the same time [64]. However, this will be a challenging task, as the flying drones with a limited battery support and single antennas will need to be replaced with UM-MIMO, which will be an important research avenue for the upcoming 6G systems. For instance, recently, THz UM-MIMO communications has been considered for a Space-Air-Ground Integrated Network (SAGIN) comprising of terrestrial, airborne and spaceborne networks [65]. With the plasmonic antenna arrays having nano-antenna spacings, it will be possible to practically implement $\mathrm{THz}$ systems onto the flying drones within miniature footprints [52].

\section{Reconfigurable Intelligent Surfaces}

Recently, novel tunable metasurfaces are referred to as Reconfigurable Intelligent Surfaces (RIS), which can be used for controlling and optimizing the wireless channel environment $[66,67,68,69]$. Generally, strong NLOS signals having specular reflections take the surfaces of the existing building infrastructures as electric mirrors, particularly at considerably miniature wavelengths across the THz band. However, RIS built from the metasurfaces and discrete element semiconductors also enables usercustomized settings. A similar RIS can sufficiently increase the $\mathrm{THz}$ signal power by reflecting the $\mathrm{THz}$ signals towards a specific direction. This can be achieved by introducing required phase shifts of the discrete elements in the RIS. In addition, a sufficiently large RIS supporting the aforementioned features can be acquired within miniature footprints at high frequencies, such as the $\mathrm{THz}$ band $[22,70,71]$. RISs have already been considered for improving the coverage performance of $\mathrm{THz}$ indoor communications at the sea level, as in [72], where the authors have proposed a suboptimal search scheme for the RIS phase shifts. Additionally, RISs have also been consi-dered for $\mathrm{THz}$ drone communications [73]. For the drone networks at the low altitude scenarios, e.g., $h_{t}=$ $100 \mathrm{~m}$ and below, up to sea level, $\mathrm{THz}$ communication ranges can be substantially extended with the aid of RISs deployed on top of buildings, roofs etc. This can also be achieved for drone-to-ground and ground-to-drone links by placing RISs near the intended user access points [14], Thus, RIS can considerably increase the coverage of a drone network e.g., drone base station, agricultural monitoring in a rural area with a limited or no terrestrial communication infrastructure. etc.

\subsection{Spectrum and interference management}

With the progression towards $6 \mathrm{G}$, exploitation of the higher frequency bands above existing sub $6 \mathrm{GHz}$ spectrum has become more appealing than ever. This will raise the need of sharing the spectrum using cognitive radio sensing with flexibility [74]. THz spectrum has been identified as a prime communication band for mobile communications within $6 \mathrm{G}$ research $[28,75]$. Also, $2 \mathrm{G}$ to $5 \mathrm{G}$ networks across the globe have been utilizing lower frequency bands, which will also be available in $6 \mathrm{G}$ networks. Therefore, various spectrum management techniques will be needed for managing the lower, mid and higher frequency bands intelligently. Here, the massive $\mathrm{THz}$ band will suffice the spectrum scarcity by assigning different frequency sub-bands to different users subject to different scenarios, mitigating the conventional issue of interference. For instance, in [76, 77], Long-User-CentralWindow (LUCW) is considered, where the farther users are allocated to the central sub-bands of a $\mathrm{THz}$ transmission window, while the the users at shorter transmission ranges are provisioned with the edge sub-bands in a window. Such an interference management can also be employed within a drone network, where a drone (e.g., drone base station) serving different users can utilize dif- ferent sub-bands each several $\mathrm{GHz}$ wide [12], supporting capacity values in the order of several $100 \mathrm{~s}$ of Gbps even under BM fading and MP fading as discussed earlier in Section 3. Nevertheless, interference in $\mathrm{THz}$ band com- munications usually occurs in dense scenarios $[78,79]$. Thus, drone networks can be deployed strategically, e.g., via sharp pencil beam-forming, to mitigate the issue of the interference [6]. 


\subsection{MAC and higher network layers}

Similar to the physical layer-related issues, various challenges also emerge across the higher protocol stack layers. To start with the link layer, new medium access control (MAC) protocols are necessary to address the unique characteristics of the $\mathrm{THz}$ band as well as DSNs. Here, availability of the massive bandwidth annihilates the basic requirement for the communicating nodes to contest for the channel. Furthermore, $\mathrm{THz}$ signals with a miniature transmit duration also diminishes the chances of collision. Also, with the razor-sharp beams used in $\mathrm{THz}$ band transmission systems, MAC design for DSNs should facilitate receiver-initiated novel transmission schemes, so that the transmitter resources are not wasted when the intended receiver is unavailable. Novel MAC protocols managing the $\mathrm{THz}$ band communications for drone networks should also involve optimization of the packet size and error control techniques in an adaptive fashion. At the network layer, novel routing techniques should be devised to provision both the traditional active nodes (for relaying) together with new passive intelligent reflecting surfaces, which can relay the incoming $\mathrm{THz}$ signals towards the intended destination node [80]. Furthermore, novel metrics of routing are needed to be devised that captures the unique channel composition on the molecular level. These novel routing metrics can include the effect of the molecular composition on the distance and altitude-dependent $\mathrm{THz}$ bandwidth [12]. Across the transport layer, with $\mathrm{THz}$ band promising communication at 100s of Gbps or even up to Tbps, network congestion will drastically increase. This will give rise to issues across the transport layer pertaining to the flow/ congestion control, also ensuring end-to-end trans- port with reliability. For instance, it is expected that the conventional Transport Control Protocol (TCP) congestion control windowing will be revised to tackle the huge traffic demands of the THz band networks [81]. In addition to adapting MAC and higher layers to work efficiently with respect to the characteristics of the $\mathrm{THz}$ channel, the potential solutions should address the mobility of drones and DSN scenarios.

\subsection{Security and privacy}

Apart from the inherent advantages of the massive bandwidth and the huge rates $\mathrm{THz}$ band can offer drone networks with mobility [22]; security and privacy also comes up as a byproduct. Various unique characteristics of the THz waves highly influence the security and privacy [82]. With the unique $\mathrm{THz}$ absorption Spectra, secure wireless communications can coexist along with THz-based detection and imaging. Massive $\mathrm{THz}$ bandwidth favors antijamming approaches. However, the performance of such $\mathrm{THz}$ systems will be environment dependent, i.e., water vapor concentration levels. Huge attenuation at THz fre- quencies ensure link secrecy, while the razor-sharp THz beams favors covert communication, at the cost of rapid coordination of the communicating $\mathrm{Tx}$ and Rx beams. In regards to the privacy aspects, $\mathrm{THz}$ communications are hard to eavesdrop from a large distance due to the huge attenuation at THz frequencies near sea level. Meanwhile, a study shows that the THz signals can be intercepted by placing an object within the razor-sharp LOS THz beam for scattering the beam towards the eavesdropper [83]. For DSNs and drone networks at lower atmospheric altitudes (typically within a few 100s of meters above sea level), secure THz ultra-broadband communications can be established with the razor-sharp THz Tx-Rx beams pointing towards each other, leveraging the drone mobility, while the LOS beam scattering as shown in [83] will become difficult to realize for the eavesdroppers. This can be highly leveraged for sensitive applications, such as in military surveillance, border patrolling, etc. To summa- rize, we have studied several open issues and research directions towards realizing THz-enabled drone networks and DSNs from the physical layer to the higher network layers dealing with security and privacy issues. A similar study has recently been provided in [6], where the $\mathrm{THz}$ wireless systems have been defined based on seven features including: 1) Quasi-optical nature, 2) THz-based architectures,

3) Coexistence with the lower frequency bands, 4) Joint communication and sensing systems, 5) Physical layer strategies, 6) Spectrum access methods, and 7) Network optimization in real time. In the following, we overview the state-of-the-artartificial intelligence and machine leaning-based solutions to the problems related to the $\mathrm{THz}$ communications, particularly for the THz-enabled drone networks, in detail.

\section{AI/ML BASED SOLUTIONS}

The advent of Artificial Intelligence (AI) in the communications paradigm has been recently surged. Various tutorials and survey works have been published within the past few years on the AI/ML implementation for wireless communications [84, 85, 86, 87]. Among various recent studies, AI has been considered as the focus of $6 \mathrm{G}$ networks $[88,89,90]$ for complementing the traditional methods. With the AI/ML technologies for Beyond 5G (B5G) systems, it will be possible to minimize/replace the existing manual network configuration management, as well as to ensure and deliver overall higher system performance with increased reliability. Moreover, communication networks will be able to adapt conveniently in real time based on the behavior of the users and the communication network. All in all, AI/ML will promise adaptive configuration and management of the communication networks by learning patterns and adapting to certain communication scenarios with flexibility, e.g., learning the communication traffic and planning in anticipa- tion [91]. A similar approach can be considered for the THz-enabled drone networks, where based on AI/ML techniques, a swarm of drones 
can adjust their respective positions optimally in order to route and maintain Tbps links among communicating drones. In what follows, we review some $\mathrm{AI} /$ ML-based approaches possibly implementable for THz-enabled drone networks for channel estimation, UM-MIMO, and Mobile edge computing.

\subsection{Channel estimation}

As discussed earlier in Section 1, first, the THz band is highly affected from absorption loss due to water vapor molecules in the atmosphere, contributing significantly to the total loss. Second, the spread loss is also massive at $\mathrm{THz}$ frequencies. Third, the $\mathrm{THz}$ band channels are nonstationary, particularly for mobile use cases i.e., hovering drones in our case, where both the Tx and Rx drones will be mobile. Hence, conventional assumptions of quasistationary or stationary channel models may not be applied to the $\mathrm{THz}$ band channels. More specifically, channel estimation over the $\mathrm{THz}$ band becomes more challenging in the drone scenarios under mobility, where precise Channel State Information (CSI) is needed, e.g., in beam-forming. Therefore, the traditional channel estimation methodologies are required to be revisited [52]. Overall, for reducing the complexity of the $\mathrm{THz}$ channel estimation, several techniques can be employed includ- ing: compressed sensing, fast channel tracking-based algorithms, etc. ML-based algorithms, in this context, can be employed for evaluating the $\mathrm{THz}$ band communication data by anticipating the $\mathrm{THz}$ signal loss in a certain unknown channel. Consequently, various AI or ML-based algorithms are applicable to the physical layer of the forth-coming 6G wireless networks for addressing the above-mentioned $\mathrm{THz}$ channel model and estimation [92, 93]. Supervised Learning (SL) [94] can aid in predicting $\mathrm{THz}$ shadowing and path loss. Moreover, SL can be employed for localization, channel estimation, interference management, etc. Employable SL models and algorithms are K-Nearest Neighbor (KNN), Support Vector Machine (SVM), feed-forward neural networks, and radial basis function neural networks, etc. Various challenges related to the $\mathrm{THz}$ channel modeling and estimation including multi-path tracking, interference mitigation, node clustering, optimized modulation, etc., can be tackled by using Unsupervised Learning (UL) techniques [95] such as: Fuzzy C-means, K-means, clustering algorithms, etc. Deep Learning (DL) (both SL and UL) can be employed in many aspects of channel modeling, such as for signal detection, and estimating Channel State Information (CSI). Techniques including Deep Neural Networks (DNNs), Recurrent Neural Networks (RNNs), Convolutional Neural Net- works (CNNs) can be anticipated as appropriate candidate DL algorithms [96]. Reinforcement Leaning (RL)[97] can be used for channel selection and tracking, iden- tification of radio bands, selection of modulation modes, etc. Appropriate RL models and techniques include Q-learning, fuzzy RL, etc. [98]. Finally, learning-based schemes for $\mathrm{THz}$ channel estimation is highly efficient particularly over higher dimensions [99]. As an example of deep kernel-based learning, a Gaussian process regression is studied in [100] for the channel estimation of a UM-MIMO multi-user system over the THz band (0.06$10 \mathrm{THz}$ ). It is to be noted here that the AI/ML techniques mentioned above are proposed for $6 \mathrm{G}$ wireless communications and networks at sea level in general. Therefore, there will be a need to tailor these AI/ML techniques specifically for the THz-enabled drone networks and DSNs keeping in view the intrinsic nature of the communicating drones i.e., mobility, energyconstrained resources etc.

\subsection{UM-MIMO}

In the realm of UM-MIMO, ML can be employed in various use cases. One instance is when an existing model is erroneous, and/or it is only a sparse approximate of the actual model. Such an instance can arise within the linear channel models, where the non-linearities induced by certain practical circumstances and hardware are neglected. Also, ML can be utilized for improving the solu- tions obtained using approximations of the linear mod-els. Another instance of ML-based solution in UM-MIMO is possible when the optimized solutions are computa- tionally expensive i.e., not feasible for the state-of-the-art hardware. Here, ML can be effectively utilized for finding suboptimal solutions having less complexity, with some obvious/ acceptable lower performance. Examples in this context include channel estimation, maximum likelihood detection, etc. Moreover, optimum spectrum utilization in UM-MIMO can be made possible using machine learning techniques [22].

\subsection{Mobile edge computing}

Mobile Edge Computing (MEC) has recently emerged as a technique for $5 \mathrm{G}$ networks, in which cloud computinglike functionalities are processed at the edges of the cellular networks [101]. MEC can equip mobile devices, such as drones with constrained processing capabilities, to hand over their processing tasks to the nearest network's edge. Conversely, within a THz-enabled DSN, mobile user equipment can offload its computationally expensive jobs to the serving drones with MEC functionalities. Low latency systems for sporadic access such as cyber-physical communication systems (a.k.a., Tactile Internet) [102] require latencies within sub-ms for controlling hovering objects (drones in our case). Such alike systems will also be a requirement for evolved industry 4.0 applications [103]. It is predicted that the transport methods over the physical layer will be linked with edge computing such as MEC, or real-time cloud computing within the vicinity of the communication network. The main objective here is to deliver resources/solutions to the evolving IoT protocols comprising of a massive number of inter-connected devices with constrained energy and storage requirements such as in drone networks, as well with some latency requirements. For overcoming these constraints, global 6G research is moving towards distributed computation techniques (MEC here) [104, 105]. 
As an example, by em- ploying AI/ML using a DNN, initial extraction of the fea- tures can be obtained using drones, which are then re- layed at the network edges for the subsequent processing. Here, a THz band can be highly leveraged by relaying massive data to the network edges for MEC. In addition, novel energy efficient MAC protocols for THz-enabled drone networks and DSNs will be required for intelligently of- floading massive computations to the network edges, also considering drone mobility effects.

\section{CONCLUSIONS}

In this paper, we have summarized major characteristics of the various possible real-world applications of the THz-enabled drone networks and DSNs. After presenting the capacity potential of the THz-enabled drone networks via numerical results considering both ideal and realistic (fading) conditions of beam misalignment fading and multipath fading, we have discussed the major research challenges and directions for THz-enabled drone networks and DSNs, from physical layer channel estimation up to higher network layers, security/privacy issues. We outline and highlight AI/ML-based approaches as promising solutions. We proclaim that $\mathrm{THz}$-enabled DSNs will be an integral part of the forthcoming 6G non-terrestrial networks. For development and validation of the $\mathrm{THz}$ band solutions across all layers, novel test beds for the real-world experiments are essential. Additionally, with the progression towards practical $\mathrm{THz}$ transceivers at sea level, novel $\mathrm{THz}$ transceivers for the drones will be required towards realizing 6G communications keeping in view the highly mobile nature of the drones, UM-MIMO given the battery limitations, and the sharp $\mathrm{THz}$ beams. Currently, such works are mostly limited to the near-THz transmission windows of $300-650 \mathrm{GHz}$, albeit, wireless systems at actual $\mathrm{THz}$ carrier frequencies $(0.1 \mathrm{THz}$ up to $10 \mathrm{THz}$ ) will be needed. Along with research directions mentioned in this paper, also further work is expected for standardizing and regulating the $\mathrm{THz}$ band.

\section{ACKNOWLEDGEMENT}

This work is supported in part by the Higher Education Commission (HEC), Government of Pakistan under the PhD scholarship program titled: HRDI Faculty Development of UESTPs Batch-V, SAP No: 50028241.

\section{REFERENCES}

[1] S. Hayat, E. Yanmaz, and R. Muzaffar, "Survey on Unmanned Aerial Vehicle Networks for Civil Applications: A Communications Viewpoint," IEEE Communications Surveys Tutorials, vol. 18, no. 4, pp. 2624-2661, 2016.

[2] E. Yanmaz, S. Yahyanejad, B. Rinner, H. Hellwagner, and C. Bettstetter, "Drone networks: Communications, coordination, and sensing," $\mathrm{Ad}$
Hoc Networks, vol. 68, pp. 1 - 15, 2018, advances in Wireless Communication and Networking for Cooperating Autonomous Systems. [Online]. Available: http://www.sciencedirect.com/ science/article/pii/S1570870517301671

[3] G. Chmaj and H. Selvaraj, "Distributed Processing Applications for UAV/drones: A Survey," in Progress in Systems Engineering, H. Selvaraj, D. Zydek, and G. Chmaj, Eds. Cham: Springer International Publishing, 2015, pp. 449-454.

[4] M. Latva-aho, "Radio Access Networking Challenges Towards 2030," in Proc. 1st International Telecommunication Union Workshop on Network 2030, New York, Oct. 2018. [Online]. Available: https://www.itu.int/en/ITU-T/ Workshops-and-Seminars/201810/Documents/ Matt_Latvaaho_Presentation.pdf

[5] Z. Zhang, Y. Xiao, Z. Ma, M. Xiao, Z. Ding, X. Lei, G. K. Karagiannidis, and P. Fan, "6G Wireless Networks: Vision, Requirements, Architecture, and Key Technologies," IEEE Vehicular Technology Magazine, vol. 14, no. 3, pp. 28-41, 2019.

[6] C. Chaccour, M. N. Soorki, W. Saad, M. Bennis, P. Popovski, and M. Debbah, "Seven Defining Features of Terahertz (THz) Wireless systems: A Fellowship of Communication and Sensing," arXiv, 2021. [Online]. Available: https://arxiv.org/abs/ 2102.07668

[7] L. Bariah, L. Mohjazi, S. Muhaidat, P. C. Sofotasios, G. K. Kurt, H. Yanikomeroglu, and O. A. Dobre, "A Prospective Look: Key Enabling Technologies, Applications and Open Research Topics in 6G Networks," IEEE Access, vol. 8, pp. 174 792-174 $820,2020$.

[8] A. A. Boulogeorgos, A. Alexiou, T. Merkle, C. Schubert, R. Elschner, A. Katsiotis, P. Stavrianos, D. Kritharidis, P. Chartsias, J. Kokkoniemi, M. Juntti, J. Lehtomaki, A. Teixeira, and F. Rodrigues, "Terahertz Technologies to Deliver Optical Network Quality of Experience in Wireless Systems Beyond 5G," IEEE Communications Magazine, vol. 56, no. 6, pp. 144-151, 2018.

[9] I. F. Akyildiz and J. M. Jornet, "Realizing UltraMassive MIMO $(1024 \times 1024)$ Communication in the (0.06-10) Terahertz band," Nano Comm. Netw., vol. 8, pp. 46-54, 2016.

[10] I. F. Akyildiz, J. M. Jornet, and C. Han, "Terahertz Band: Next Frontier for Wireless Communications," Physical Communication, vol. 12, p. 16-32, Sep. 2014.

[11] A. Saeed, O. Gurbuz, and M. A. Akkas, "Terahertz Communications at Various Atmospheric 
Altitudes," Physical Communication, vol. 41, p. 101113, 2020.

[12] A. Saeed, O. Gurbuz, A. O. Bicen, and M. A. Akkas, "Variable-Bandwidth Model and Capacity Analysis for Aerial Communications in the Terahertz Band," IEEE Journal on Selected Areas in Communications, pp. 1-1, 2021.

[13] V. Petrov, D. Moltchanov, M. Komar, A. Antonov, P. Kustarev, S. Rakheja, and Y. Koucheryavy, "Terahertz Band Intra-Chip Communications: Can Wireless Links Scale Modern x86 CPUs?" IEEE Access, vol. 5, pp. 6095-6109, 2017.

[14] J. M. Jornet and I. F. Akyildiz, "Channel Modeling and Capacity Analysis for Electromagnetic Wireless Nanonetworks in the Terahertz Band," IEEE Trans. Wireless Communications, vol. 10, no. 10, pp. 3211-3221, 2011. [Online]. Available: https: //doi.org/10.1109/TWC.2011.081011.100545

[15] S. Mumtaz, J. Jornet, J. Aulin, W. Gerstacker, X. Dong, and b. ai, "Terahertz Communication for Vehicular Networks," IEEE Transactions on Vehicular Technology, vol. 66, pp. 5617-5625, 072017.

[16] S. Krishna Moorthy and Z. Guan, "Beam Learning in Mmwave/THz-band Drone Networks Under InFlight Mobility Uncertainties," IEEE Transactions on Mobile Computing, pp. 1-1, 2020.

[17] Z. Guan and T. Kulkarni, "On the Effects of Mobility Uncertainties on Wireless Communications between Flying Drones in the mmWave/THz Bands," in IEEE INFOCOM 2019 - IEEE Conference on Computer Communications Workshops (INFOCOM WK-SHPS), 2019, pp. 768-773.

[18] X. Wang, P. Wang, M. Ding, Z. Lin, F. Lin, B. Vucetic, and L. Hanzo, "Performance Analysis of Terahertz Unmanned Aerial Vehicular Networks," IEEE Transactions on Vehicular Technology, vol. 69, no. 12, pp. 16330-16335, 2020.

[19] R. Mendrzik, D. Cabric, and G. Bauch, "Error Bounds for Terahertz MIMO Positioning of Swarm UAVs for Distributed Sensing," in 2018 IEEE International Conference on Communications Workshops (ICC Workshops), 2018, pp. 1-6.

[20] G. Ding, Q. Wu, L. Zhang, Y. Lin, T. A. Tsiftsis, and Y. Yao, "An Amateur Drone Surveillance System Based on the Cognitive Internet of Things," IEEE Communications Magazine, vol. 56, no. 1, pp. 2935, 2018.

[21] Z. Sun, P. Wang, M. C. Vuran, M. A. Al-Rodhaan, A. M. Al-Dhelaan, and I. F. Akyildiz, "BorderSense: Border Patrol through Advanced Wireless Sensor Networks," Ad Hoc Networks, vol. 9, no. 3, pp. 468-477, 2011.
[22] H. Sarieddeen, N. Saeed, T. Y. Al-Naffouri, and M. Alouini, "Next Generation Terahertz Communications: A Rendezvous of Sensing, Imaging, and Localization," IEEE Communications Magazine, vol. 58, no. 5, pp. 69-75, 2020 .

[23] S. Ergun and S. Sonmez, "Terahertz Technology For Military Applications," Journal of Military and Information Science, vol. 3, 012015.

[24] J. Federici, B. Schulkin, F. Huang, D. Gary, R. Barat, F. Oliveira, and D. Zimdars, "Thz Imaging and Sensing for Security Applications - Explosives, Weapons and Drugs," Semiconductor Science and Technology, vol. 20, p. S266, 062005.

[25] M. Erdelj, E. Natalizio, K. R. Chowdhury, and I. F. Akyildiz, "Help from the Sky: Leveraging UAVs for Disaster Management," IEEE Pervasive Computing, vol. 16, no. 1, pp. 24-32, 2017.

[26] T. Long, M. Ozger, O. Cetinkaya, and O. B. Akan, "Energy Neutral Internet of Drones," IEEE Communications Magazine, vol. 56, no. 1, pp. 2228, 2018.

[27] N. Shimizu, H.-J. Song, Y. Kado, T. Furuta, A. Wakatsuki, and Y. Muramoto, "Gas Detection Using Terahertz Waves," vol. 7, 032009.

[28] T. S. Rappaport, Y. Xing, O. Kanhere, S. Ju, A. Madanayake, S. Mandal, A. Alkhateeb, and G. C. Trichopoulos, "Wireless Communications and Applications Above $100 \mathrm{GHz}$ : Opportunities and Challenges for 6G and Beyond," IEEE Access, vol. 7, pp. 78 729-78 757, 2019.

[29] A. Fotouhi, M. Ding, and M. Hassan, "Flying Drone Base Stations for Macro Hotspots," IEEE Access, vol. 6, pp. 19530-19539, 2018.

[30] X. Wang, H. Zhang, Y. Tian, and V. C. M. Leung, "Modeling and Analysis of Aerial Base StationAssisted Cellular Networks in Finite Areas Under LoS and NLoS Propagation," IEEE Transactions on Wireless Communications, vol. 17, no. 10, pp. 69857000, 2018.

[31] R. Singh and D. Sicker, "Reliable THz Communications for Outdoor based Applications- Use Cases and Methods," in 2020 IEEE 17th Annual Consumer Communications Networking Conference (CCNC), 2020, pp. 1-4.

[32] S. Garcia Sanchez, S. Mohanti, D. Jaisinghani, and K. R. Chowdhury, "Millimeter-wave Base Stations in the Sky: An Experimental Study of UAV-toGround Communications," IEEE Transactions on Mobile Computing, pp. 1-1, 2020.

[33] 3GPP, "Tsg.90-e plenaries," Release 17, December 122020. 
[34] J. Angjo, I. Shayea, M. Ergen, H. Mohamad, A. Alhammadi, and Y. I. Daradkeh, "Handover Management of Drones in Future Mobile Networks: 6G Technologies," IEEE Access, vol. 9, pp. 12 803-12 823, 2021.

[35] W. Saad, M. Bennis, and M. Chen, "A Vision of 6G Wireless Systems: Applications, Trends, Technologies, and Open Research Problems," IEEE Network, vol. 34, no. 3, pp. 134-142, 2020.

[36] I. Bor-Yaliniz and H. Yanikomeroglu, "The New Frontier in RAN Heterogeneity: Multi-Tier DroneCells," IEEE Communications Magazine, vol. 54, no. 11 , pp. $48-55,2016$.

[37] M. Mirahsan, R. Schoenen, and H. Yanikomeroglu, "HetHetNets: Heterogeneous Traffic Distribution in Heterogeneous Wireless Cellular Networks," IEEE Journal on Selected Areas in Communications, vol. 33, no. 10, pp. 2252-2265, 2015.

[38] A. A. Boulogeorgos, E. N. Papasotiriou, and A. Alexiou, "Analytical performance assessment of thz wireless systems," IEEE Access, vol. 7, pp. $11436-$ $11453,2019$.

[39] S.A. Clough , M.W. Shephard, E.J. Mlawer , J.S. Delamere, M.J. Iacono, K. Cady-Pereira , S. Boukabara and P.D. Brown, "Atmospheric Radiative Transfer Modeling: a Summary of the AER Codes," Journal of Quantitative Spectroscopy and Radiative Transfer, vol. 91, no. 2, pp. 233-244, 2005. [On-line]. Available: http:// www.sciencedirect.com/science/article/pii/ S0022407304002158

[40] S. A. Clough, M. J. Iacono, and J.-L. Moncet, "Line-by-Line Calculations of Atmospheric Fluxes and Cooling Rates: Application to Water Vapor," Journal of Geophysical Research: Atmospheres, vol. 97, no. D14, pp. 15 761-15 785, 1992. [Online]. Available: https://agupubs.onlinelibrary. wiley.com/doi/abs/10.1029/92JD01419

[41] P. Zhou, X. Fang, Y. Fang, R. He, Y. Long, and G. Huang, "Beam Management and Self-Healing for mmWave UAV Mesh Networks," IEEE Transactions on Vehicular Technology, vol. 68, no. 2, pp. 17181732, 2019.

[42] M. T. Dabiri, H. Safi, S. Parsaeefard, and W. Saad, "Analytical Channel Models for Millimeter Wave UAV Networks Under Hovering Fluctuations," IEEE Transactions on Wireless Communications, vol. 19, no. 4, pp. 2868-2883, 2020.

[43] A. A. Farid and S. Hranilovic, "Outage Capacity Optimization for Free-Space Optical Links With Pointing Errors," Journal of Lightwave Technology, vol. 25, no. 7, pp. 1702-1710, 2007.
[44] M. D. Yacoub, "The $\alpha-\mu$ distribution: A Physical Fading Model for the Stacy Distribution," IEEE Transactions on Vehicular Technology, vol. 56, no. 1, pp. 27-34, 2007.

[45] M. Stojanovic and J. Preisig, "Underwater acoustic communication channels: Propagation models and statistical characterization," IEEE Communications Magazine, vol. 47, no. 1, pp. 84-89, January 2009.

[46] A. Fotouhi, M. Ding, and M. Hassan, "Flying Drone Base Stations for Macro Hotspots," IEEE Access, vol. 6, pp. 19 530-19539, 2018.

[47] R. Singh and D. Sicker, "Beyond 5G: THz Spectrum Futures and Implications for Wireless Communication," International Telecommunications Society (ITS), 30th European Regional ITS Conference, Helsinki 2019 205213, June 2019. [Online]. Available: https://ideas.repec.org/p/ zbw/itse19/ 205213.html

[48] G. Castellanos, M. Deruyck, L. Martens, and W. Joseph, "Performance Evaluation of Direct-Link Backhaul for UAV-Aided Emergency Networks," Sensors, vol. 19, no. 15, 2019.

[49] A. Goldsmith, Wireless Communications. New York, NY, USA: Cambridge University Press, 2005.

[50] Y. Xing and T. S. Rappaport, “Terahertz Wireless Communications: Co-Sharing for Terrestrial and Satellite Systems Above 100 GHz," arXiv, 2021. [Online]. Available: https://arxiv.org/abs/2103. 00604

[51] C. Han and I. F. Akyildiz, "Distance-Aware MultiCarrier (DAMC) Modulation in Terahertz Band Communication," in 2014 IEEE International Conference on Communications (ICC), 2014, pp. 54615467.

[52] H. Sarieddeen, M.-S. Alouini, and T. Y. Al-Naffouri, "An Overview of Signal Processing Techniques for Terahertz Communications," arXiv, 2020. [Online]. Available: https://arxiv.org/abs/2005.13176

[53] H. Elayan, O. Amin, B. Shihada, R. M. Shubair, and M. Alouini, "Terahertz Band: The Last Piece of RF Spectrum Puzzle for Communication Systems," IEEE Open Journal of the Communications Society, vol. 1, pp. 1-32, 2020.

[54] R. C. Daniels and R. W. Heath, "60 GHz Wireless Communications: Emerging Requirements and Design Recommendations," IEEE Vehicular Technology Magazine, vol. 2, no. 3, pp. 41-50, 2007.

[55] 3GPP, "Study on New Radio Access Technology (Release 14)," 3GPP TR 38.801, 2017. 
[56] Y. Medjahdi, S. Traverso, R. Gerzaguet, H. Shaïek, R. Zayani, D. Demmer, R. Zakaria, J. Doré, M. Ben Mabrouk, D. Le Ruyet, Y. Louët, and D. Roviras, “On the Road to 5G: Comparative Study of Physi- cal Layer in MTC Context," IEEE Access, vol. 5, pp. 26 556-26 581, 2017.

[57] C. Han, A. O. Bicen, and I. F. Akyildiz, "MultiWideband Waveform Design for Distance-Adaptive Wireless Communications in the Terahertz Band," IEEE Transactions on Signal Processing, vol. 64, no. 4, pp. 910-922, Feb 2016.

[58] J. M. Jornet and I. F. Akyildiz, "Femtosecond-Long Pulse-Based Modulation for Terahertz Band Communication in Nanonetworks," IEEE Transactions on Communications, vol. 62, no. 5, pp. 1742-1754, 2014.

[59] Z. Hossain and J. M. Jornet, "Hierarchical Bandwidth Modulation for Ultra-Broadband Terahertz Communications," in ICC 2019 - 2019 IEEE International Conference on Communications (ICC), 2019, pp. 1-7.

[60] L. Dai, B. Wang, Z. Ding, Z. Wang, S. Chen, and L. Hanzo, "A Survey of Non-Orthogonal Multiple Access for 5G," IEEE Communications Surveys Tutorials, vol. 20, no. 3, pp. 2294-2323, 2018.

[61] X. Zhang, C. Han, and X. Wang, "Joint BeamformingPower-Bandwidth Allocation in Terahertz NOMA Networks," in 2019 16th Annual IEEE International Conference on Sensing, Communication, and Networking (SECON), 2019, pp. 1-9.

[62] A. A. Boulogeorgos, E. N. Papasotiriou, and A. Alexiou, "A Distance and Bandwidth Dependent Adaptive Modulation Scheme for $\mathrm{THz}$ Communications," in 2018 IEEE 19th International Workshop on Sig- nal Processing Advances in Wireless Communica-tions (SPAWC), 2018, pp. 1-5.

[63] I. Akyildiz and J. Jornet, "Realizing Ultra-Massive MIMO (1024 x 1024) Communication in the (0.0610) Terahertz Band," Nano Communication Networks, vol. 8, pp. 46-54, 62016.

[64] P. Chandhar and E. G. Larsson, "Massive MIMO for Connectivity With Drones: Case Studies and Future Directions," IEEE Access, vol. 7, pp. $94676-$ $94691,2019$.

[65] A. Liao, Z. Gao, D. Wang, H. Wang, H. Yin, D. W. K. Ng, and M.-S. Alouini, "Terahertz Ultra-Massive MIMOBased Aeronautical Communications in Space-AirGround Integrated networks," IEEE Journal on Selected Areas in Communications, vol. 39, no. 6, pp. 1741-1767, 2021.
[66] 0. Tsilipakos, A. C. Tasolamprou, A. Pitilakis, F. Liu, X. Wang, M. S. Mirmoosa, D. C. Tzarouchis, S. Abadal, H. Taghvaee, C. Liaskos, A. Tsioliaridou, J. Georgiou, A. Cabellos-Aparicio, E. Alarc $\tilde{A}^{3} n$, S. Ioannidis, A. Pitsillides, I. F. Akyildiz, N. V. Kantartzis, E. N. Economou, C. M. Soukoulis, M. Kafesaki, and S. Tretyakov, "Toward Intelligent Metasurfaces: The Progress from Globally Tunable Metasurfaces to Software-Defined Metasurfaces with an Embedded Network of Controllers," Advanced Optical Materials, vol. 8, no. 17, p. 2000783, 2020. [Online]. Available: https://onlinelibrary. wiley.com/doi/abs/10.1002/adom.202000783

[67] E. Basar, M. Di Renzo, J. De Rosny, M. Debbah, M. S. Alouini, and R. Zhang, "Wireless Communications Through Reconfigurable Intelligent Surfaces," IEEE Access, vol. 7, pp. 116 753-116 773, 2019.

[68] M. Di Renzo, M. Debbah, D.-T. Phan-Huy, A. Zappone, M. S. Alouini, C. Yuen, V. Sciancalepore, G. Alexandropoulos, J. Hoydis, H. Gacanin, J. D. Rosny, A. Bounceur, G. Lerosey, and M. Fink, "Smart Radio Environments Empowered by Reconfigurable AI Meta-Surfaces: An Idea Whose Time Has Come," EURASIP Journal on Wireless Communications and Networking, no. 129, 2019.

[69] R. Alghamdi, R. Alhadrami, D. Alhothali, H. Almorad, A. Faisal, S. Helal, R. Shalabi, R. Asfour, N. Hammad, A. Shams, N. Saeed, H. Dahrouj, T. Y. Al-Naffouri, and M. S. Alouini, "Intelligent Surfaces for 6G Wireless Networks: A Survey of Optimization and Performance Analysis Techniques," IEEE Access, vol. 8, pp. 202 795-202 818, 2020.

[70] C. Chaccour, M. N. Soorki, W. Saad, M. Bennis, and P. Popovski, "Risk-Based Optimization of Virtual Reality over Terahertz Reconfigurable Intelligent Surfaces," in ICC 2020 - 2020 IEEE International Conference on Communications (ICC), 2020, pp. 1-6.

[71] A.-A. A. Boulogeorgos and A. Alexiou, "Coverage Analysis of Reconfigurable Intelligent Surface Assisted THz Wireless Systems," IEEE Open Journal of Vehicular Technology, vol. 2, pp. 94-110, 2021.

[72] X. Ma, Z. Chen, W. Chen, Y. Chi, Z. Li, C. Han, and Q. Wen, "Intelligent Reflecting Surface Enhanced Indoor Terahertz Communication Systems," Nano Communication Networks, vol. 24, p. 100284, 2020.

[73] N. Abuzainab, M. Alrabeiah, A. Alkhateeb, and Y. E. Sagduyu, "Deep Learning for THz Drones with Flying Intelligent Surfaces: Beam and Handoff Prediction," arXiv, 2021. [Online]. Available: https: //arxiv.org/abs/2102.11222

[74] M. Matinmikko-Blue, S. Yrj lä, and P. Ahokangas, "Spectrum Management in the 6G Era: The Role of Regulation and Spectrum Sharing," in 2020 2nd 6G Wireless Summit (6G SUMMIT), 2020, pp. 1-5. 
[75] B. Aazhang, P. Ahokangas, H. Alves, M.-S. Alouini, J. Beek, H. Benn, M. Bennis, J. Belfiore, E. Strinati, F. Chen, K. Chang, F. Clazzer, S. Dizit, D. Kwon, M. Giordiani, W. Haselmayr, J. Haapola, E. Hardouin, E. Harjula, and P. Zhu, Key Drivers and Research Challenges for $6 G$ Ubiquitous Wireless Intelligence (White Paper), 092019.

[76] C. Han and I. F. Akyildiz, "Distance-Aware Bandwidth-Adaptive Resource Allocation for Wireless Systems in the Terahertz Band," IEEE Transactions on Terahertz Science and Technology, vol. 6, no. 4, pp. 541-553, 2016.

[77] M. Yu, A. Tang, X. Wang, and C. Han, "Joint Scheduling and Power Allocation for 6G Terahertz Mesh Networks," in 2020 International Conference on Computing, Networking and Communications (ICNC), 2020, pp. 631-635.

[78] C. Chaccour, M. N. Soorki, W. Saad, M. Bennis, and P. Popovski, "Can Terahertz Provide HighRate Reliable Low Latency Communications for Wireless VR?" arXiv, 2021. [Online]. Available: https://arxiv.org/abs/2005.00536

[79] V. Petrov, D. Moltchanov, and Y. Koucheryavy, "Interference and SINR in Dense Terahertz Networks," in 2015 IEEE 82nd Vehicular Technology Conference (VTC2015-Fall), 2015, pp.1-5.

[80] Q. Wu, S. Zhang, B. Zheng, C. You, and R. Zhang, "Intelligent Reflecting Surface Aided Wireless Communications: A Tutorial," IEEE Transactions on Communications, pp. 1-1, 2021.

[81] NetWorld2020, "Strategic Research and Innovation Agenda 2021-27: Smart Networks in the Context of NGI," European Technology Platform NetWorld2020, 2018.

[82] R. Singh and D. Sicker, "Thz Communications - A Boon and/or Bane for Security, Privacy, and National Security," SSRN Electronic Journal, 012020.

[83] J. Ma, R. Shrestha, J. Adelberg, C.-Y. Yeh, Z. Hossain, E. Knightly, J. Jornet, and D. Mittleman, "Security and Eavesdropping in Terahertz Wireless Links," Nature, vol. 563, 112018.

[84] X. Wang, Y. Han, V. C. M. Leung, D. Niyato, X. Yan, and X. Chen, "Convergence of Edge Computing and Deep Learning: A Comprehensive Survey," IEEE Communications Surveys Tutorials, vol. 22, no. 2, pp. 869-904, 2020.

[85] N. C. Luong, D. T. Hoang, S. Gong, D. Niyato, P. Wang, E. Liang, and D. I. Kim, "Applications of Deep Reinforcement Learning in Communications and Networking: A Survey," IEEE Communications Surveys Tutorials, vol. 21, no. 4, pp. 3133-3174, 2019.
[86] Y. Sun, M. Peng, Y. Zhou, Y. Huang, and S. Mao, "Application of Machine Learning in Wireless Networks: Key Techniques and Open Issues," IEEE Communications Surveys Tutorials, vol. 21, no. 4, pp. 3072-3108, 2019.

[87] J. Jagannath, N. Polosky, A. Jagannath, F. Restuccia, and T. Melodia, "Machine Learning for Wireless Communications in the Internet of Things: A Comprehensive Survey," Ad Hoc Networks, vol. 93, p. 101913, 2019. [Online]. Available: https://www.sciencedirect.com/science/ article/pii/S1570870519300812

[88] L. Zhang, Y. Liang, and D. Niyato, "6G Visions: Mobile Ultra-Broadband, Super Internet-of-Things, and Artificial Intelligence," China Communications, vol. 16, no. 8, pp. 1-14, 2019.

[89] G. Gui, M. Liu, F. Tang, N. Kato, and F. Adachi, “6G: Opening new horizons for integration of comfort, security, and intelligence," IEEE Wireless Communications, vol. 27, no. 5, pp. 126-132, 2020.

[90] K. B. Letaief, W. Chen, Y. Shi, J. Zhang, and Y. A. Zhang, "The Roadmap to 6G: AI Empowered Wireless Networks," IEEE Communications Magazine, vol. 57, no. 8, pp. 84-90, 2019.

[91] C.-X. Wang, M. D. Renzo, S. Stanczak, S. Wang, and E. G. Larsson, "Artificial intelligence enabled wireless networking for $5 \mathrm{G}$ and beyond: Recent advances and future challenges," IEEE Wireless Communications, vol. 27, no. 1, pp. 16-23, 2020.

[92] J. Du, C. Jiang, J. Wang, Y. Ren, and M. Debbah, "Machine Learning for 6G Wireless Networks: Carrying Forward Enhanced Bandwidth, Massive Access, and Ultrareliable/Low-Latency Service," IEEE Vehicular Technology Magazine, vol. 15, no. 4, pp. 122-134, 2020.

[93] A. Zappone, M. Di Renzo, and M. Debbah, "Wireless Networks design in the Era of Deep Learning: Model-based, AI-Based, or Both?" IEEE Transactions on Communications, vol. 67, no. 10, pp. 73317376, 2019.

[94] M. E. Morocho-Cayamcela, H. Lee, and W. Lim, "Machine Learning for 5G/B5G Mobile and Wireless Communications: Potential, Limitations, and $\mathrm{Fu}-$ ture Directions," IEEE Access, vol. 7, pp. $137184-$ $137206,2019$.

[95] R. Nikbakht, A. Jonsson, and A. Lozano, "Unsupervised Learning for Parametric Optimization," IEEE Communications Letters, vol. 25, no. 3, pp. 678681, 2021. 
[96] L. Dai, R. Jiao, F. Adachi, H. V. Poor, and L. Hanzo, "Deep Learning for Wireless Communications: An Emerging Interdisciplinary Paradigm," IEEE Wireless Communications, vol. 27, no. 4, pp. 133-139, 2020.

[97] R. S. Sutton and A. G. Barto, Reinforcement Learning: An Introduction. MIT Press, 2017.

[98] Y. S. Nasir and D. Guo, "Multi-Agent Deep Reinforcement Learning for Dynamic Power Allocation in Wireless Networks," IEEE Journal on Selected Areas in Communications, vol. 37, no. 10, pp. 2239-2250, 2019.

[99] H. He, R. Wang, W. Jin, S. Jin, C.-K. Wen, and G. Y. Li, "Beamspace Channel Estimation for Wideband Millimeter-Wave MIMO: A Model-Driven Unsupervised Learning Approach," arXiv, 2021. [Online]. Available: https://arxiv.org/abs/2006.16628

[100] S. Nie and I. F. Akyildiz, "Deep Kernel LearningBased Channel Estimation in Ultra-Massive MIMO Communications at 0.06-10 thz," in 2019 IEEE Globecom Workshops (GC Wkshps), 2019, pp. 1-6.

[101] A. A. Al-Habob, A. Ibrahim, O. A. Dobre, and A. G. Armada, "Collision-Free Sequential Task Offloading for Mobile Edge Computing," IEEE Communications Letters, vol. 24, no. 1, pp. 71-75, 2020.

[102] G. Fettweis, "The Tactile Internet: Applications and Challenges," Vehicular Technology Magazine, IEEE, vol. 9, pp. 64-70, 032014.

[103] H. Lasi, P. Fettke, H.-G. Kemper, T. Feld, and M. Hoffmann, "Industry 4.0," Business and Information Systems Engineering, vol. 6, pp. 239-242, 082014.

[104] K. B. Letaief, W. Chen, Y. Shi, J. Zhang, and Y.-J. A. Zhang, "The Roadmap to 6G: AI Empowered Wireless Networks," IEEE Communications Magazine, vol. 57, no. 8, pp. 84-90, 2019.

[105] Y. Mao, C. You, J. Zhang, K. Huang, and K. B. Letaief, "A Survey on Mobile Edge Computing: The Communication Perspective," IEEE Communications Surveys Tutorials, vol. 19, no. 4, pp. 23222358, 2017.

\section{AUTHORS}

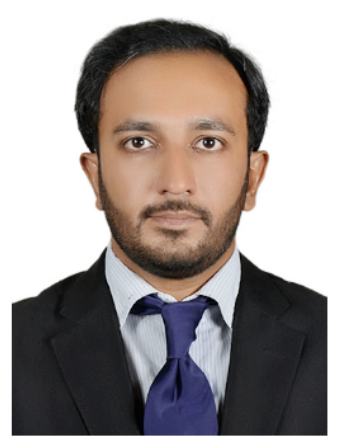

Dr. Akhtar Saeed received his B.E. degree in telecommunications engineering at U.I.T Hamdard University in 2010 and M.E. degree in telecommunications engineering at NED University of Engineering and Technology in 2014 . He received his Ph.D. degree in electronics engineering from Sabanci University in 2021 under the supervision of Dr. Ozgur Gurbuz and co-supervision of Dr. Mustafa Alper Akkaş. His research interests include terahertz communications at various atmospheric altitudes, millimeter wave communications, wireless sensor networks and full duplex communications.

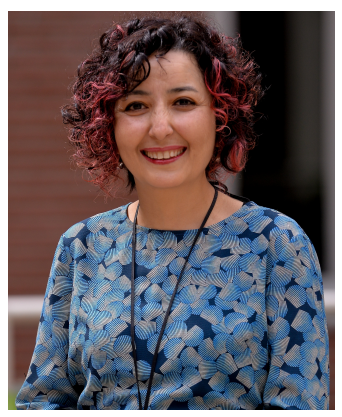

Dr. Ozgur Gurbuz received her B.S. and M.S. degrees in electrical and electronics engineering at Middle East Technical University, in 1992 and 1995, respectively. She received her Ph.D. degree in electrical and computer engineering from Georgia Institute of Technology in 2000. From 2000 until 2002 she worked as a researcher and sys-

tems/algorithms engineer for Cisco Systems, in Wireless Access and Wireless Networking Business Units. As of September 2002, Dr. Gurbuz joined the Faculty of Engineering and Natural Sciences at Sabanci University, where she is serving as a professor. Her research interests remain in the field of wireless communications and networks, specifically design of link and higher layer network algorithms/protocols for emerging physical layer techniques, including full-duplex communication, cooperative communication, MIMO, smart antennas. Recently, she has been working on full-duplex communications, terahertz communications and applications of machine learning in wireless communications/networks. 


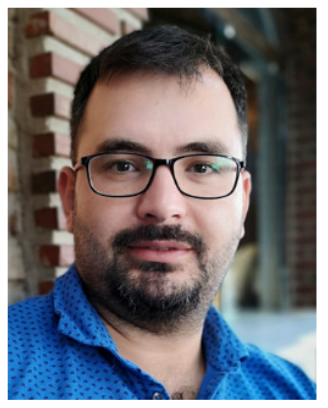

Dr. Mustafa Alper Akkaş received his B.S. degree in electrical and electronics engineering from Erciyes University in 2006. He received his Ph.D. degree in the Department of Electrical and Electronics Engineering at Ege University in 2014. Currently, he is an associate professor at the Department of Computer Engineering at Bolu Abant Izzet Baysal University. His research interests include terahertzband communication networks, channel modeling, wireless underground communication networks, internet of things and intra-body wireless nanosensor networks. He was a visiting student at Georgia Institute of Technology, in the Broadband Wireless Networking Lab under the supervision of Prof. Ian F. Akyildiz from September 2011 to May 2012.

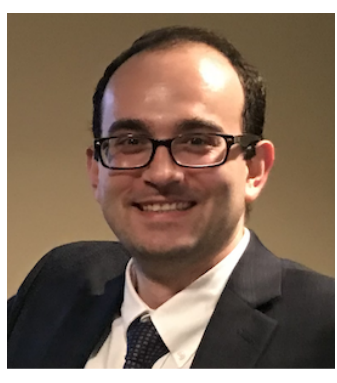

Dr. Ahmet Ozan Bicen received B.Sc. degree in electrical and electronics engineering from Middle East Technical University, Ankara, Turkey, in 2010, M.Sc. degree in electrical and electronics engineering from Koc University, Istanbul, Turkey, in 2012, and Ph.D. degree in electrical and computer engineering from the Georgia Institute of Technology, Atlanta, GA, USA, in 2016. He was a postdoctoral researcher in the same department in between 2016-2018. He was an Assistant Professor in the Faculty of Engineering and Natural Sciences, Sabanci University, Istanbul, Turkey, in between 2019-2021. His current research interests include statistical signal processing, molecular communication, and computational physiology. 